PAPER

\section{Hyperons in neutron star matter within relativistic mean-field models}

To cite this article: M Oertel et al 2015 J. Phys. G: Nucl. Part. Phys. 42075202

View the article online for updates and enhancements.
Related content

- MANY-BODY FORCES IN THE
EQUATION OF STATE OF HYPERONIC
MATTER
R. O. Gomes, V. Dexheimer, S. Schramm
et al.
- Compact stars and the symmetry energy
Constana Providência, Rafael Cavagnoli,
Debora P Menezes et al.
- Nonlinear
llona Bednarek and Ryszard Manka

\section{Recent citations}

- The attribute of rotational profile to the
$\frac{\text { hyperon puzzle in the prediction of }}{\text { heaviest compact star }}$
M. Bhuyan et al
- Equations of state for supernovae and
$\frac{\text { compact stars }}{\text { M. Oertel } \text { et al }}$
- Examination of strangeness instabilities
$\frac{\text { and effects of strange meson couplings in }}{\text { dense strange hadronic matter and }}$
$\frac{\text { compact stars }}{\text { James R. Torres et al }}$




\title{
Hyperons in neutron star matter within relativistic mean-field models
}

\author{
M Oertel ${ }^{1,5}$, C Providência $^{2}$, F Gulminelli ${ }^{3}$ and Ad R Raduta ${ }^{4}$ \\ ${ }^{1}$ LUTH, CNRS, Observatoire de Paris, Université Paris Diderot, 5 place Jules Janssen, \\ 92195 Meudon, France \\ ${ }^{2}$ Centro de Física Computacional, Department of Physics, University of Coimbra, \\ P-3004-056 Coimbra, Portugal \\ ${ }^{3}$ CNRS/ENSICAEN/LPC/Université de Caen Basse Normandie, UMR6534, F-14050 \\ Caen Cedex, France \\ ${ }^{4}$ IFIN-HH, Bucharest-Magurele, POB-MG6, Romania \\ E-mail: micaela.oertel@obspm.fr, cp@teor.fis.uc.pt, gulminelli@1pccaen.in2p3.fr and \\ araduta@nipne.ro
}

Received 15 December 2014, revised 24 February 2015

Accepted for publication 17 March 2015

Published 8 June 2015

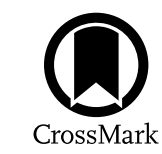

\begin{abstract}
Since the discovery of neutron stars with masses around $2 M_{\odot}$ the composition of matter in the central part of these massive stars has been intensively discussed. Within this paper we will (re)investigate the question of the appearance of hyperons. To that end we will perform an extensive parameter study within relativistic mean field models. We will show that it is possible to obtain high mass neutron stars with (i) a substantial amount of hyperons, (ii) radii of $12-13 \mathrm{~km}$ for the canonical mass of $1.4 M_{\odot}$, and (iii) a spinodal instability at the onset of hyperons. The results depend strongly on the interaction in the hyperon-hyperon channels, on which only very little information is available from terrestrial experiments up to now.
\end{abstract}

Keywords: neutron stars, equation of state, thermodynamic instabilities

(Some figures may appear in colour only in the online journal)

\section{Introduction}

With the purpose of better understanding the dynamics of core-collapse supernova and the observed neutron star characteristics, considerable theoretical effort has been undertaken in

5 Author to whom any correspondence should be addressed. 
recent years concerning the modelization of the equations of state $(\mathrm{EoS})$ of cold dense matter with some extensions to finite temperature.

If we admit that hyperonic and deconfined quark matter could exist in the inner core of neutron stars, a complete understanding of its composition is far from being achieved. Concerning hyperons, simple energetic considerations suggest that they should be present at high density [1]. However, in the standard picture, the opening of hyperon degrees of freedom leads to a considerable softening of the EoS [1], which in turns leads to maximum neutron star masses smaller than the highest observed values [2, 3]. This puzzling situation could be circumvented by a very early deconfinement transition [4], or by the population of other baryonic states such as $\Delta$-baryons pushing the hyperon onset to higher densities. The latter, however, only replaces the hyperon puzzle by a $\Delta$ puzzle [5] unless a phase transition to quark matter is simultaneously invoked.

Without calling upon such a transition, it has been shown within phenomenological density functional models that the observed neutron star masses simply imply that the hyperon-hyperon $(Y Y)$ and hyperon-nucleon $(Y N)$ couplings must be much more repulsive at high density than presently assumed (e.g. [6-17]). The general agreement is, however, that the price to pay for this additional repulsion is a very low strangeness content of neutron stars [10].

In microscopic calculations the missing repulsion for hyperons is more difficult to obtain. Naturally, one would expect it to arise from three-body forces. But, using a microscopic model based on the Brueckner-Hartree-Fock (BHF) approach, the authors of [18] found that even phenomenologically adding a three-body force was not enough to allow for the existence of stars which are massive enough to be compatible with observations. On the other hand, in recent calculations using an auxiliary field diffusion Monte Carlo method (AFQMC) $[19,20]$, the authors found that a sufficiently strong repulsive three-body force, constrained by the systematics of separation energies in a series of hypernuclei, can produce a stiff enough equation of state of hyperneutron matter that satisfies the $2 M_{\odot}$ constraint, even if a strong model dependence due to the phenomenological nature of the hyperon two- and three-body force is apparent. With the stiffest interaction model studied in [20], the repulsive part of the energy functional is important enough to shift the onset of hyperons to densities above 0.56 $\mathrm{fm}^{-3}$ [20], confirming the finding from density functional models that the solution to the hyperon puzzle could simply be a reduced hyperon content of neutron stars.

However, it should be pointed out that within the BHF approach (purely nuclear) stellar matter becomes superluminous at the center of massive neutron stars when hyperons are not included in the calculation [18]. This problem is inherent to the non-relativistic nature of BHF calculations and could appear in any non-relativistic approach, e.g. AFQMC. It is therefore important to check the effect of repulsive interactions in the hyperon channels on a larger variety of models, particularly relativistic models where the sound speed correctly behaves at any density. At this point it should be mentioned that recent relativistic Dirac-BruecknerHartree-Fock (DBHF) calculations [21], including automatically part of the three-body forces, reproduce hyperonic neutron stars with two solar masses, but with a nuclear EoS that is either too stiff or does not give enough binding, in contradiction with known properties of symmetric nuclear matter at saturation. In this context, relativistic mean field models, which have proven a powerful tool in the description of nuclei, hypernuclei and dense (hyper) nuclear matter, can give useful insight and the dependence of the neutron star structure on the hyperonic couplings can be studied. In particular, we will show in this paper that the $2 M_{\odot}$ constraint can be reached even with a non-negligible fraction of hyperons in the star's core. There is still some uncertainty due to the poor present knowledge of the hyperonic interaction 
and we would like to stress the importance of further, more stringent constraints from ab initio models and/or experimental data.

Another argument put forward against hyperons is that the strong repulsion needed leads to an overpressure close to nuclear saturation density, which is inconsistent with microphysical constraints derived by [38]. This overpressure is also associated with large radii for neutron stars, larger than those suggested for intermediate mass neutron stars by recent observations. In many models with hyperons compatible with the neutron star mass constraint, see e.g. [8, 10], indeed relatively high radii of about $14 \mathrm{~km}$ for a nonrotating spherical neutron star with the canonical mass of $1.4 M_{\odot}$ are obtained. This can, however, not be a general argument since there are some examples with lower radii $[7,13,15]$.

In addition, the generic presence of attractive and repulsive couplings suggests the existence, in a model-independent manner, of a phase transition involving strangeness. A detailed study of the phase diagram of dense baryonic matter was recently undertaken in [2325] within a non-relativistic mean-field model based on phenomenological functionals. It was shown that under these assumptions first- and second- order phase transitions exist, and are expected to be explored under the strangeness equilibrium condition characteristic of stellar matter. In [26] such a phase transition has been discussed for relativistic mean field models, but within a model with very strong $Y Y$ attraction.

Here we are interested in (a) examining in which region of parameter space within relativistic mean field (RMF) models a first order phase transition from purely nuclear to hyperonic matter could exist and if the existence of such a phase transition is compatible with experimental and observational data, (b) if it is possible to obtain high mass neutron stars with a considerable amount of hyperons and (c) finding additional support for hyperonic EoS with radii of $12-13 \mathrm{~km}$ for canonical $1.4 M_{\odot}$ neutron stars and maximum masses in agreement with observations.

We will work at zero temperature throughout the whole paper. The paper is organized as follows. In section 2 we discuss the model applied and the setup for the hyperonic interactions. We explain the procedure to detect thermodynamic instabilities in section 3 . In section 4.1 we present results for neutron star matter containing nucleons, electrons and $\Lambda$ hyperons. We extent the discussion to the full baryonic octet in section 4.2 and we close the paper with a summary of the results in section 5 .

\section{The model}

The literature on phenomenological RMF models including hyperons is large and many different versions exist (see e.g. [27]), including either non-linear couplings or densitydependent ones of baryons to the meson fields mediating the interaction. Let us stress at this point that, although they are generally called meson fields, these fields are purely phenomenological and only serve to describe the interaction without any relation with existing meson fields, except for the quantum numbers which give the names for the corresponding RMF meson fields. The Lagrangian of the model can be written in the following form 


$$
\begin{aligned}
\mathcal{L}= & \sum_{j \in \mathcal{B}} \bar{\psi}_{j}\left(i \gamma_{\mu} \partial^{\mu}-m_{j}+g_{\sigma j} \sigma+g_{\sigma^{* j}} \sigma^{*}\right. \\
& \left.+g_{\delta j} \vec{\delta} \cdot \vec{I}_{j}-g_{\omega j} \gamma_{\mu} \omega^{\mu}-g_{\phi j} \gamma_{\mu} \phi^{\mu}-g_{\rho j} \gamma_{\mu} \vec{\rho}^{\mu} \cdot \vec{I}_{j}\right) \psi_{j} \\
& +\frac{1}{2}\left(\partial_{\mu} \sigma \partial^{\mu} \sigma-m_{\sigma}^{2} \sigma^{2}\right)-\frac{1}{3} g_{2} \sigma^{3}-\frac{1}{4} g_{3} \sigma^{4} \\
& +\frac{1}{2}\left(\partial_{\mu} \sigma^{*} \partial^{\mu} \sigma^{*}-m_{\sigma^{*}}^{2} \sigma^{*^{2}}\right) \\
& +\frac{1}{2}\left(\partial_{\mu} \vec{\delta} \partial^{\mu} \vec{\delta}-m_{\delta}^{2} \vec{\delta}^{2}\right) \\
& -\frac{1}{4} W_{\mu \nu}^{\dagger} W^{\mu \nu}-\frac{1}{4} P_{\mu \nu}^{\dagger} P^{\mu \nu}-\frac{1}{4} \vec{R}_{\mu \nu}^{\dagger} \cdot \vec{R}^{\mu \nu} \\
& +\frac{1}{2} m_{\omega}^{2} \omega_{\mu} \omega^{\mu}+\frac{1}{4} c_{3}\left(\omega_{\mu} \omega^{\mu}\right)^{2} \\
& +\frac{1}{2} m_{\phi}^{2} \phi_{\mu} \phi^{\mu}+\frac{1}{2} m_{\rho}^{2} \vec{\rho}_{\mu} \cdot \vec{\rho}^{\mu}
\end{aligned}
$$

where $\psi_{j}$ denotes the field of baryon $j$, and $W_{\mu \nu}, P_{\mu \nu}, \vec{R}_{\mu \nu}$ are the vector meson field tensors of the form

$$
V^{\mu \nu}=\partial^{\mu} V^{\nu}-\partial^{\nu} V^{\mu} .
$$

$\sigma, \sigma^{*}$ are scalar-isoscalar meson fields, coupling to all baryons $(\sigma)$ and to strange baryons $\left(\sigma^{*}\right)$, respectively. $\vec{\delta}$ induces a scalar-isovector coupling.

In the mean field approximation, the meson fields are replaced by their respective meanfield expectation values, which are given in uniform matter as

$$
\begin{aligned}
& m_{\sigma}^{2} \bar{\sigma}+g_{2} \bar{\sigma}^{2}+g_{3} \bar{\sigma}^{3}=\sum_{i \in B} g_{\sigma i} n_{i}^{s} \\
& m_{\sigma^{*}}^{2} \bar{\sigma}^{*}=\sum_{i \in B} g_{\sigma^{*} i} n_{i}^{s} \\
& m_{\delta}^{2} \bar{\delta}=\sum_{i \in B} g_{\delta i} t_{3 i} n_{i}^{s} \\
& m_{\omega}^{2} \bar{\omega}+c_{3} \bar{\omega}^{3}=\sum_{i \in B} g_{\omega i} n_{i} \\
& m_{\phi}^{2} \bar{\phi}=\sum_{i \in B} g_{\phi i} n_{i} \\
& m_{\rho}^{2} \bar{\rho}=\sum_{i \in B} g_{\rho i} t_{3 i} n_{i},
\end{aligned}
$$

where $\bar{\delta}=\left\langle\delta_{3}\right\rangle, \bar{\rho}=\left\langle\rho_{3}^{0}\right\rangle, \bar{\omega}=\left\langle\omega^{0}\right\rangle, \bar{\phi}=\left\langle\phi^{0}\right\rangle$, and $t_{3 i}$ represents the third component of isospin of baryon $i$ with the convention that $t_{3 p}=1 / 2$. The scalar density of baryon $i$ is given by

$$
n_{i}^{s}=\left\langle\bar{\psi}_{i} \psi_{i}\right\rangle=\frac{1}{\pi^{2}} \int_{0}^{k_{F i}} k^{2} \frac{M_{i}^{*}}{\sqrt{k^{2}+M_{i}^{* 2}}} \mathrm{~d} k
$$


and the number density by

$$
n_{i}=\left\langle\bar{\psi}_{i} \gamma^{0} \psi_{i}\right\rangle=\frac{1}{\pi^{2}} \int_{0}^{k_{F i}} k^{2} \mathrm{~d} k=\frac{k_{F i}^{3}}{3 \pi^{2}} .
$$

The effective baryon mass $M_{i}^{*}$ depends on the scalar mean fields as

$$
M_{i}^{*}=M_{i}-g_{\sigma i} \bar{\sigma}-g_{\sigma^{*} i} \bar{\sigma}^{*}-g_{\delta i} t_{3 i} \bar{\delta},
$$

and the effective chemical potentials, $\left(\mu_{i}^{*}\right)^{2}=\left(M_{i}^{*}\right)^{2}+k_{F i}^{2}$, are related to the chemical potentials via

$$
\mu_{i}^{*}=\mu_{i}-g_{\omega i} \bar{\omega}-g_{\rho i} t_{3 i} \bar{\rho}-g_{\phi i} \bar{\phi}-\Sigma_{0}^{R} .
$$

The rearrangement term

$$
\begin{aligned}
\Sigma_{0}^{R}= & \sum_{j \in B}\left(\frac{\partial g_{\omega j}}{\partial n_{j}} \bar{\omega} n_{j}+t_{3 j} \frac{\partial g_{\rho j}}{\partial n_{j}} \bar{\rho} n_{j}+\frac{\partial g_{\phi j}}{\partial n_{j}} \bar{\phi} n_{j}\right. \\
& \left.-\frac{\partial g_{\sigma j}}{\partial n_{j}} \bar{\sigma} n_{j}^{s}-\frac{\partial g_{\sigma^{* j}}}{\partial n_{j}} \bar{\sigma}^{*} n_{j}^{s}-t_{3 j} \frac{\partial g_{\delta j}}{\partial n_{j}} \bar{\delta} n_{j}^{s}\right)
\end{aligned}
$$

is present in density dependent models to ensure thermodynamic consistency.

For the present study we will limit ourselves to two non-linear models, GM1 [28] and TM1-2 [29], and one density-dependent model, DDH $\delta$ [30, 31]. The two non-linear ones have been chosen among the large number of existing models because they are widely used and they have been obtained assuming very different strategies for determining the parameters: GM1 has been adjusted to nuclear saturation properties imposing a certain effective mass and incompressibility, whereas TM1 has been fitted to ground state properties of nuclei and at high densities to DBHF calculations. For the GM1 parametrization, $c_{3}=0$, and the $\delta$ field is absent in GM1 and TM1-2. The density-dependent models assume $g_{2}=g_{3}=c_{3}=0$ (no non-linear terms) and the following density dependence of the couplings is used within DDH $\delta$

$$
g_{i}\left(n_{B}\right)=g_{i}\left(n_{0}\right) h_{i}(x), \quad x=n_{B} / n_{0},
$$

with $n_{0}$ denoting nuclear matter saturation density and

$$
h_{i}(x)=a_{i} \frac{1+b_{i}\left(x+d_{i}\right)^{2}}{1+c_{i}\left(x+d_{i}\right)^{2}}
$$

for all isoscalar couplings and

$$
h_{i}(x)=a_{i} \exp \left[-b_{i}(x-1)\right]-c_{i}\left(x-d_{i}\right) .
$$

for the isovector ones. The parameter values for GM1 can be found, e.g., in [32], table III, for TM1-2 in [29], table I, and for the DDH $\delta$ model in [30], table II. The EoS of homogeneous symmetric nuclear matter for parametrization TM1-2 is shown in figure 1 of [29]. It has the same properties as TM1 [33] at and below saturation density, but it is stiffer at suprasaturation densities, still within the constraints imposed by heavy-ion flow [34]. However, within this parametrization the slope of the symmetry energy at saturation is very large, $L=110 \mathrm{MeV}$. Since the radius of compact stars is very sensitive to $L$ [35-37], we will consider a modified version with $L=55 \mathrm{MeV}$ [29], too, introducing a non-linear $\omega-\rho$ term as in [35]. The resulting properties of homogeneous symmetric nuclear matter are listed in 
Table 1. Nuclear matter properties of the models considered in this study for symmetric nuclear matter at saturation, except for the last column where the pressure of $\beta$-equilibrated neutron star matter at zero temperature and a baryon number density of $n_{0}$ is given.

\begin{tabular}{lcccccc}
\hline & $\begin{array}{c}K \\
(\mathrm{MeV})\end{array}$ & $\begin{array}{c}E_{\text {sym }} \\
(\mathrm{MeV})\end{array}$ & $\begin{array}{c}n_{0} \\
(\mathrm{MeV})\end{array}$ & $\begin{array}{c}B \\
\left(\mathrm{fm}^{-3}\right)\end{array}$ & $\begin{array}{c}L \\
(\mathrm{MeV})\end{array}$ & $\begin{array}{c}P\left(n_{0}\right) \\
\left(\mathrm{MeV} \mathrm{fm}^{-3}\right)\end{array}$ \\
\hline GM1 & 300 & 32.5 & 0.153 & 16.3 & 94 & 4.06 \\
TM1-2 & 281 & 36.9 & 0.145 & 16.3 & $110 / 55$ & $4.38 / 2.43$ \\
DDH $\delta$ & 240 & 25.1 & 0.153 & 16.3 & 44 & 2.56 \\
\hline
\end{tabular}

table 1 for all parametrizations employed within the present paper. In the same table we also include the value of the pressure of $\beta$-equilibrated cold neutron star matter at $n_{0}$ for reference. According to [38], where a microscopic neutron matter calculation in the framework of a chiral effective field theory together with available information on symmetric nuclear matter have been used to build the EoS of stellar matter, this value should lie in the range

$$
1.8 \lesssim P\left(n_{0}\right) \lesssim 3.0 \mathrm{MeV} \mathrm{fm}^{-3} .
$$

This range of pressures is the result of a quite restrictive allowed region for the symmetry energy $(29.7-33.5 \mathrm{MeV})$ and its slope $L(32.4-57 \mathrm{MeV})$ at saturation.

\subsection{Setup for the hyperonic interaction}

The wealth of nuclear data allows one to constrain the nuclear interaction parameters within reasonable ranges, whereas this is not the case for hyperons, where data are scarce. These leaves some freedom in adjusting the interaction parameters for the hyperonic sector.

Many recent works, see e.g. $[10,15,32]$, use a procedure inspired by the symmetries of the baryon octet to express the individual isoscalar vector meson-baryon couplings in terms of $g_{\omega N}$ and a few additional parameters [39] as follows

$$
\begin{aligned}
& \frac{g_{\omega \Lambda}}{g_{\omega N}}=\frac{1-\frac{2 z}{\sqrt{3}}(1-\alpha) \tan \theta}{1-\frac{z}{\sqrt{3}}(1-4 \alpha) \tan \theta}, \\
& \frac{g_{\phi \Lambda}}{g_{\omega N}}=-\frac{\tan \theta+\frac{2 z}{\sqrt{3}}(1-\alpha)}{1-\frac{z}{\sqrt{3}}(1-4 \alpha) \tan \theta}, \\
& \frac{g_{\omega \Xi}}{g_{\omega N}}=\frac{1-\frac{z}{\sqrt{3}}(1+2 \alpha) \tan \theta}{1-\frac{z}{\sqrt{3}}(1-4 \alpha) \tan \theta}, \\
& \frac{\tan \theta+\frac{z}{\sqrt{3}}(1+2 \alpha)}{g_{\phi \Xi}}=-\frac{z}{1-\frac{z}{\sqrt{3}}(1-4 \alpha) \tan \theta},
\end{aligned}
$$




$$
\begin{aligned}
& \frac{g_{\omega \Sigma}}{g_{\omega N}}=\frac{1+\frac{2 z}{\sqrt{3}}(1-\alpha) \tan \theta}{1-\frac{z}{\sqrt{3}}(1-4 \alpha) \tan \theta} \\
& \frac{g_{\phi \Sigma}}{g_{\omega N}}=\frac{-\tan \theta+\frac{2 z}{\sqrt{3}}(1-\alpha)}{1-\frac{z}{\sqrt{3}}(1-4 \alpha) \tan \theta} \\
& \frac{g_{\phi N}}{g_{\omega N}}=-\frac{\tan \theta+\frac{z}{\sqrt{3}}(1-4 \alpha)}{1-\frac{z}{\sqrt{3}}(1-4 \alpha) \tan \theta}
\end{aligned}
$$

The parameter $\alpha$ thereby determines the ratio of symmetric coupling of the baryons to the vector meson octet $\left(D\right.$-term) and the antisymmetric coupling $\left(F\right.$-term), and $g_{1}$ and $g_{8}$ are the coupling constants for coupling of baryons to the vector meson singlet and octet, respectively. $\theta$ is the mixing angle of $\omega$ - and $\phi$-mesons with the corresponding singlet and octet states, and $z=g_{8} / g_{1}$. As commonly assumed, in what follows we will take $\tan \theta=1 / \sqrt{2}$, corresponding to ideal mixing and $\alpha=1$. In the literature, $S U(6)$-symmetry is mostly imposed to fix the couplings, i.e. $z=1 / \sqrt{6}$, and only recent studies in view of the observation of high mass neutron stars have relaxed this assumption, for example [10, 14, 32].

It should be pointed out that if $z \neq 1 / \sqrt{6}$, the $\phi$ meson couples to nucleons and it has to be included upon determining the parameters of the model that reproduce nuclear saturation properties, see [10], equation (12). This means that we have readjusted $g_{\omega N}$ upon varying $z$, throughout all the calculations respecting the symmetry constraints on the isoscalar vector meson couplings given by equation (18).

In the isovector sector symmetry arguments are not used since this would lead to contradictions with the observed nuclear symmetry energy. $g_{\rho N}$ is therefore left as a free parameter, adjusted to the desired value of the symmetry energy, and the remaining nonvanishing isovector vector couplings are all equal, the isospin symmetry being taken into account through the isospin operator $t_{3 i}$, see equation (12),

$$
g_{\rho \Lambda}=0, \quad g_{\rho N}=g_{\rho \Xi}=g_{\rho \Sigma} \text {. }
$$

For the scalar sector, in [13] a symmetry inspired procedure is discussed and tested against the constraints imposed by hypernuclear data. Here, as done in e.g. $[10,15]$, we will directly use the information from hypernuclear data on hyperonic single-particle mean field potentials to constrain the coupling constants. The potential for particle $j$ in $k$-particle matter is given by

$$
U_{j}^{(k)}\left(n_{k}\right)=M_{j}^{*}-M_{j}+\mu_{j}-\mu_{j}^{*}
$$

Based on data on $\Lambda$-hypernuclei produced in $\left(\pi^{+}, K^{+}\right)$reactions, the presently accepted value of the $\Lambda$-potential in symmetric nuclear matter at saturation density, $U_{\Lambda}^{(N)}\left(n_{0}\right)$, is $\approx-30 \mathrm{MeV}$ [40]. $U_{\Xi}^{(N)}\left(n_{0}\right)$ is attractive, too, with a value of $\approx-14--18 \mathrm{MeV}$, based on missing mass measurements in the $\left(K^{-}, K^{+}\right)$reaction on carbon [41]. The situation of $U_{\Sigma}^{(N)}\left(n_{0}\right)$ is ambiguous. On the one hand $\left(\pi^{-}, K^{+}\right)$reactions on medium-to-heavy nuclei point to a repulsive potential of up to $100 \mathrm{MeV}$ [42]. On the other hand, the observation of a ${ }_{\Sigma}^{4} \mathrm{He}$ bound state in a ${ }^{4} \mathrm{He}\left(K^{-}, \pi^{-}\right)$reaction [43] pleads in favor of an attractive potential. Following the 
above procedure to fix the vector coupling constants, the couplings of hyperons to $\sigma$ are then adjusted to reproduce the hyperon potentials in symmetric nuclear matter.

Very few multi-hyperon exotic nuclei data exist so far and all of them correspond to double- $\Lambda$ light nuclei. Data on the bond energy can be reinterpreted in terms of the $\Lambda$ potential in $\Lambda$ matter at the average density of $\Lambda$ inside those nuclei [44]. Mean-field calculations suggest that in light nuclei (from He to $\mathrm{C}$ ) the average $\Lambda$ density is close to one fifth of the saturation density [44, 45]. Therefore, we take as an indicative value for $U_{\Lambda}^{(\Lambda)}\left(n_{0} / 5\right)$ the experimental value of $\Delta B_{\Lambda}^{\Lambda}$. Data on ${ }_{\Lambda \Lambda}^{10} \mathrm{Be}$ and ${ }_{\Lambda \Lambda}^{13} \mathrm{~B}$ then suggest $U_{\Lambda}^{(\Lambda)}\left(n_{0} / 5\right) \approx-5 \mathrm{MeV}$ [46] while ${ }_{\Lambda \Lambda}^{6} \mathrm{He}$ data point toward a higher value of $U_{\Lambda}^{(\Lambda)}\left(n_{0} / 5\right) \approx-0.67 \mathrm{MeV}[47,48]$.

We will use here as a guideline that experimental data point towards $U_{\Lambda}^{(\Lambda)}\left(n_{0} / 5\right)>-5$ $\mathrm{MeV}$. For the other potentials it is often assumed that in isospin symmetric $\Xi$ - and $\Sigma$-matter, $U_{\Xi}^{(\Xi)}\left(n_{0}\right) \approx 2 U_{\Lambda}^{(\Lambda)}\left(n_{0} / 2\right)$ and $U_{\Sigma}^{(\Sigma)}\left(n_{0}\right) \approx U_{\Lambda}^{(\Lambda)}\left(n_{0} / 2\right)$ [49] based on theoretical estimates. In view of the only weakly attractive $\Lambda \Lambda$-potential and the uncertainties on other hyperonhyperon $(Y Y)$ potentials, often $\sigma^{*}$ is neglected (see e.g. [8, 10, 15]).

In a first step we will use the procedure described above and study the dependence of the results upon variations of the couplings to $\sigma^{*}$ and $z$. The symmetry arguments in the isoscalar vector sector are, however, not very compelling. They are based on the naive quark model for hadrons in vacuum and it is known that this model is too simple. The interactions of the baryon octet in vacuum respect an approximate $S U(3)$-flavor symmetry, but symmetry breaking effects are large. Since we are dealing here with an effective model for interacting particles in matter, without any input about symmetry breaking effects in dense matter, there is no reason to assume any flavor $S U(3)$-symmetry for the effective interaction. In addition, the approach is inconsistent in the sense that symmetry constraints are imposed only for the isoscalar vector couplings with other prescriptions for the other channels, see also the discussion in [14] on this point. By the way, in the vector-isovector channel, a strict application of this procedure would lead to severe problems with the observed nuclear symmetry energy [10]. Therefore, in a second step, we will only keep the $N Y$-potentials at some given value and vary the different coupling parameters freely. Note that for these parametrizations, where we have relaxed the symmetry constraints, we have considered $g_{\phi N}=0$ and the nuclear matter properties, see table 1 , remain unaffected by a modification of the hyperonic couplings.

\section{Thermodynamic instabilities}

The existence of a first order phase transition can be spotted by analyzing the curvature of a thermodynamic potential in terms of extensive variables, indicating the presence of a spinodal instability related to the phase transition. The unstable region is thereby recognized by a negative curvature. This convexity analysis has often been employed, among others for the phase transition to hyperonic matter in [23-25] or for the neutron-proton system [50-52]. At zero temperature, the adequate thermodynamic potential is given by the total energy density, $\varepsilon\left(\left\{n_{i}\right\}\right)$, with as variables the number densities corresponding to good quantum numbers ${ }^{6}$.

In the present case, assuming equilibrium with respect to strong and electromagnetic interaction, for purely baryonic matter the good quantum numbers are baryon number, strangeness and charge with densities $n_{B}, n_{S}$ and $n_{Q}$, respectively. Since we are interested in

${ }^{6}$ Let us stress that for the stability analysis we assume that the time-scale of potential density fluctuations is such that equilibrium with respect to strong interaction is always maintained. This allows one to reduce the ninedimensional space of all densities to a three-dimensional one. In this sense our study corresponds to the semi-frozen case of [55]. 
neutron star matter, we have to impose electrical charge neutrality and add leptonic degrees of freedom with lepton number as an additional degree of freedom. Due to the strict electrical neutrality condition, charge is no longer a good degree of freedom and the system remains three-dimensional [24,53], see also [54], in terms of the number densities $n_{B}, n_{S}$ and $n_{L}$.

Stability can now be checked by analyzing the eigen-values of the curvature matrix, $C_{i j}=\partial^{2} \varepsilon\left(\left\{n_{l}\right\}_{l=\{i, j, k\}}\right) / \partial n_{i} \partial n_{j}$, where $i, j, k=B, S, L$. The number of negative eigenvalues corresponds to the number of directions in density space, in which density fluctuations get spontaneously and exponentially amplified in order to achieve phase separation. In all our studies at most one negative eigenvalue has been found.

Muons could be included in the analysis, since they are a priori present in neutron star matter. Neglecting neutrino oscillations, they would add another dimension, corresponding to conserved muon lepton number. However, as leptons are treated as an ideal gas, they change the stability analysis only through the electrical charge neutrality constraint. Therefore, including muons in addition to electrons does not qualitatively change the results. The quantitative modifications are so small that we have decided to neglect muons for simplicity.

Exploring the complete three-dimensional space $n_{B}, n_{S}, n_{L}$ is a very demanding task. Since we are mainly interested in neutron star matter, we will restrict our investigation to the case of strangeness changing weak equilibrium, i.e. $\mu_{S}=0$. In addition we will assume $\beta$ equilibrium. We will thereby consider that a neutron star older than several minutes is cold enough such that neutrinos can freely leave the system. This means that their chemical potential is zero, i.e. the chemical potentials associated with (electron) lepton number vanish, $\mu_{L}=0$.

Let us stress that, although we restrict the study to a line in the three-dimensional density space, the stability analysis remains three-dimensional: at every point on the $\mu_{S}=0, \mu_{L}=0$ -line, the curvature matrix tests fluctuations in three directions, meaning that we do not assume weak equilibrium to be maintained throughout the fluctuations, see [55], too.

\section{Results and discussion}

The results presented below assume $U_{\Lambda}^{(N)}\left(n_{0}\right)=-28 \mathrm{MeV}, U_{\Xi}^{(N)}\left(n_{0}\right)=-18 \mathrm{MeV}$, and $U_{\Sigma}^{(N)}\left(n_{0}\right)=30 \mathrm{MeV}$ unless otherwise stated. To study the parameter dependence, we will vary the different coupling constants keeping the nuclear matter properties of the different models constant.

\subsection{Neutron star matter with $\Lambda$-hyperons}

4.1.1. Stability analysis. We will start the discussion with the simple case of matter containing nucleons, $\Lambda$-hyperons and electrons. Although not completely realistic, it is instructive since it allows one to see trends in the parameter dependence. As mentioned earlier, in a first step we will follow the procedure proposed in [10] to vary the coupling constants, see section 2.1 for details. The values of the isoscalar vector couplings are then determined by the value of the parameter $z$ and the couplings to $\sigma$ are obtained from the hyperon potentials in nuclear matter. We will thereby vary $z$ between $z=0$ and $z=1 / \sqrt{6}$, the $S U(6)$ value, since for higher values of $z$ it becomes more and more difficult to obtain neutron star maximum masses in agreement with observations, see [10] and section 4.1.2.

In figure 1 the minimal eigenvalue of the curvature matrix, $c_{\min }$, is displayed as a function of baryon density for neutron star matter within GM1 and DDH $\delta$ models, varying $z$ and $g_{\sigma^{*} \Lambda}$. The kink in the curves indicates the respective threshold density for the onset of $\Lambda$-hyperons. 

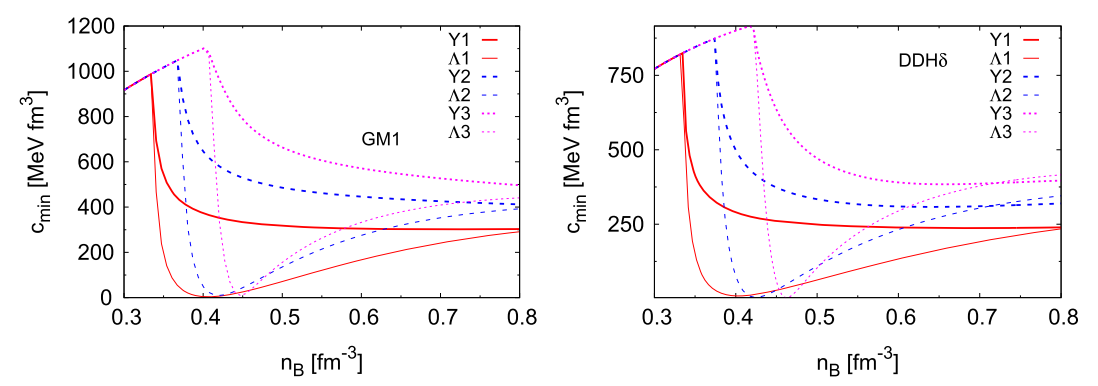

Figure 1. Smallest eigenvalue of the curvature matrix of the energy density as a function of baryon number density for neutron star matter with $R_{\sigma^{*} i}=g_{\sigma^{*} i} / g_{\sigma N}$. The GM1 and DDH $\delta$ parameter sets have been employed. Only $\Lambda$-hyperons are considered. To test the parameter dependence we have respected the symmetry constraints in the isoscalar vector sector, see equation (18). The couplings to $\sigma$ are adjusted to the hyperon potentials in nuclear matter and the $\sigma^{*}$ are varied, see section 2.1 for more details.

The lower value of $g_{\sigma^{*} \Lambda}$ has thereby been chosen such that the value $U_{\Lambda}^{(\Lambda)}\left(n_{0} / 5\right)=-5 \mathrm{MeV}$ is reproduced, which corresponds to the strongest attraction compatible with present experimental data ${ }^{7}$. Although, after the onset of $\Lambda$-hyperons, $c_{\min }$ decreases with increasing $z$, no instability is found in this case. Upon increasing $g_{\sigma_{*}^{*} \Lambda}, c_{\min }$ further decreases and for $g_{\sigma^{*} \Lambda}>g_{\text {crit }}$ a first order phase transition in neutron star matter can be observed.

At the same time, increasing the value of $g_{\sigma^{*} \Lambda}$ renders the $\Lambda \Lambda$ interaction more attractive at low densities. The values of $U_{\Lambda}^{(\Lambda)}\left(n_{0} / 5\right)$ are given for $g_{\sigma^{*} \Lambda}=g_{\text {crit }}$ in table 2 for comparison and the respective density dependence is shown in figure 2 . The present results suggest that a first order phase transition to $n p \Lambda+e$ matter can occur in RMF models, too. The price to pay is a very strong $\Lambda-\Lambda$ attraction, which is in contradiction with the actual experimental information. In the following we use the definition $R_{\sigma^{*} i}=g_{\sigma^{*} i} / g_{\sigma N}$.

Relaxing the symmetry conditions, see equation (18), for the variation of the isoscalar vector coupling constants leads to essentially the same conclusion on the stability of neutron star matter with $\Lambda$-hyperons: an instability shows up for strongly attractive $\Lambda-\Lambda$ interactions. The value of $U_{\Lambda}^{(\Lambda)}\left(n_{0} / 5\right)$ for which the instability sets in depends only very weakly on the values of the isoscalar vector couplings and is $U_{\Lambda}^{(\Lambda)}\left(n_{0} / 5\right) \approx-40 \mathrm{MeV}$ within the DDH $\delta$ model and $U_{\Lambda}^{(\Lambda)}\left(n_{0} / 5\right) \approx-30 \mathrm{MeV}$ within GM1.

It is interesting to observe that these results are very different from what is obtained in the non-relativistic framework [25]. In that study the parameter space associated to an instability is very large and includes the present hypernuclear experimental constraints. Within the present models, we arrive at the opposite conclusion. We cannot exclude that the different functional forms associated to the energy density in the relativistic and non-relativistic framework might be at the origin of this discrepancy, since it has been observed in the past that non-relativistic functionals often present unphysical instabilities [56]. An alternative explanation might be the qualitatively different behavior of the hyperon-hyperon potentials, which in both approaches are fully phenomenological. In particular the minimum of those potentials systematically occurs below saturation density in the Skyrme functionals [25] for the considered stiffness coefficients, while in the RMF models we have analyzed, it

7 Please note that for vanishing $g_{\sigma^{*} \Lambda}$, as always assumed in the recent literature, in most cases no attraction at all in the $\Lambda$-potential is observed. 

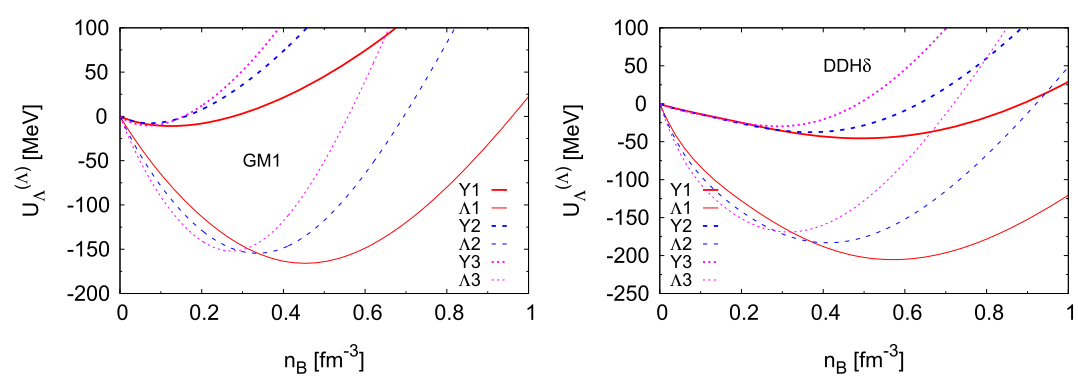

Figure 2. The $\Lambda$ single particle potential in $\Lambda$-matter as a function of baryon density. The GM1 (left) and DDH $\delta$ (right) parameter sets have been employed. The setup is the same as in figure 1.

Table 2. Summary of results respecting the symmetry arguments for the isoscalar vector couplings allowing for $\Lambda$-hyperons as the only hyperons. $R_{1.4}$ denotes the radius of a non-rotating star with $M=1.4 M_{\odot}$ and $R_{\max }$ is the radius at maximum mass. The values of $R_{\sigma^{*} \Lambda}$ for models $\Lambda n$ thereby correspond to the critical values of these coupling constants for the onset of an instability in the $\Lambda$-channel, and models denoted as $Y n$ do not present any instability. Please note that the value of $-7 \mathrm{MeV}$ for $U_{\Lambda}^{(\Lambda)}\left(n_{0} / 5\right)$ for GM1 Y3 is the highest that can be obtained within the model and the given parameter set.

\begin{tabular}{lcccccc}
\hline Model & $R_{\sigma^{*} \Lambda}$ & $z$ & $\begin{array}{c}M_{\max } \\
\left(M_{\odot}\right)\end{array}$ & $\begin{array}{c}R_{1.4} \\
(\mathrm{~km})\end{array}$ & $\begin{array}{c}R_{\max } \\
(\mathrm{km})\end{array}$ & $\begin{array}{c}U_{\Lambda}^{(\Lambda)}\left(n_{0} / 5\right) \\
(\mathrm{MeV})\end{array}$ \\
\hline $\mathrm{GM} 1$ & & & & & & \\
$Y 1$ & 0.45 & 0.41 & 1.99 & 13.8 & 12.0 & -5 \\
$\Lambda 1$ & 0.98 & 0.41 & 1.70 & 13.8 & 11.1 & -22 \\
& & & & & & \\
$Y 2$ & 0.2 & 0.2 & 2.22 & 13.8 & 12.0 & -5 \\
$\Lambda 2$ & 1.02 & 0.2 & 2.03 & 13.8 & 11.2 & -28 \\
$Y 3$ & 0 & 0. & 2.32 & 13.8 & 12.0 & -7 \\
$\Lambda 3$ & 1.08 & 0. & 2.22 & 13.8 & 11.5 & -34 \\
$\mathrm{DDH} \delta$ & & & & & & \\
$Y 1$ & 0.61 & 0.41 & 1.71 & 12.7 & 10.4 & -5 \\
$\Lambda 1$ & 1.07 & 0.41 & 1.58 & 10.5 & 9.0 & -34 \\
& & & & & & \\
$Y 2$ & 0.5 & 0.2 & 1.93 & 12.7 & 10.8 & -5 \\
$\Lambda 2$ & 1.08 & 0.2 & 1.83 & 12.7 & 9.8 & -39 \\
$Y 3$ & 0.4 & 0. & 2.06 & 12.7 & 11.1 & -5 \\
$\Lambda 3$ & 1.1 & 0. & 1.99 & 12.7 & 10.5 & -44 \\
\hline
\end{tabular}

systematically occurs above saturation. In particular, in RMF models the scalar fields saturate at large densities. The role of the scalar isoscalar meson, and scalar densities on the properties of RMF models has been discussed several times in the past, see [57, 58].

Since the instability at high baryonic density seems to be strongly correlated with the $N Y$ and $Y Y$ potentials at low hyperonic densities, there is hope in confronting the different 

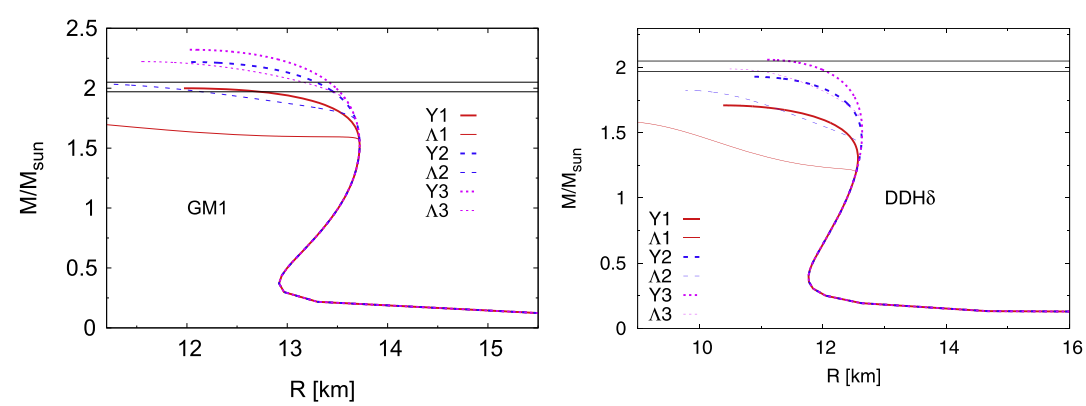

Figure 3. Mass-radius relation for non-rotating spherical neutron stars. The GM1 (left) and $\mathrm{DDH} \delta$ (right) parameter sets have been employed. The setup is the same as in figure 1. The two horizontal lines indicate the mass of PSR J0348 + 0432, $2.01 \pm 0.04 M_{\odot}$.

functional forms with new more extensive experimental hypernuclear data to solve the ambiguity. This perspective is left for future work.

4.1.2. Neutron star masses and radii. In order to obtain neutron star masses and radii we solve the Tolman-Oppenheimer-Volkoff (TOV) equations [59] for hydrostatic equilibrium of a non-rotating (spherical) star in general relativity with the different EoS. The different EoS have been supplemented at low densities with the crust EoS from Baym, Pethick and Sutherland [60]. The results for the mass-radius relation are displayed in figure 3 for GM1 (left) and $\mathrm{DDH} \delta$ (right) following the symmetry inspired procedure. The two values of $g_{\sigma^{*} \Lambda}$ for each value of $z$ thereby correspond to the cases discussed above: the one leading to the canonical value of $U_{\Lambda}^{(\Lambda)}\left(n_{0} / 5\right)=-5 \mathrm{MeV}$ and the one corresponding to the critical value for the onset of an instability. Qualitatively the results look very similar in both models.

Concerning the maximum mass associated with the different EoS, it is obvious that decreasing $z$, the maximum mass increases. This finding is not new, see [10], and is explained by the fact that a smaller $z$ leads to an interaction with stronger repulsion at high densities. Similarly, increasing $g_{\sigma^{*} \Lambda}$ renders the interaction more attractive and lowers therefore the maximum mass. In both models, maximum masses compatible with the recent observations of neutron stars with masses of $1.97 \pm 0.04$ [2] and $2.01 \pm 0.04$ [3] can be obtained. The effect of a nonzero coupling to $\sigma^{*}$ is to reduce the allowed parameter range in $z$ and smaller values of $z$ are required to obtain a high enough maximum mass. The weak attraction suggested by experimental data still allows for a wide range in $z$, whereas the strong attraction leading to an instability reduces the allowed range in $z$ considerably.

The latter conclusion can be softened relaxing the symmetry constraints on the isoscalar vector couplings, see section 4.2 where several examples are shown with acceptable neutron star masses and, at the same time, leading to the onset of an instability.

It has been claimed that the strong repulsion needed within RMF models as well in the hyperonic sector as for the purely nuclear part to obtain neutron stars compatible with recent mass measurements and containing hyperons would lead always to very large radii and that there would be a tension with recent radius determinations, see for instance [61]. Let us stress at this point that the radius determinations are difficult and that they are presently far from being as reliable as the mass observations from [2,3]. The main problem is that the extraction of radii from observations is much more model-dependent than the above-mentioned mass determinations, see e.g. [62], where a reanalysis gives a radius of $9.0_{-4}^{+2.9} \mathrm{~km}$ instead of $6.6_{1.1}^{+1.2}$ $\mathrm{km}$ [61] for the same object, a neutron star in NGC6397. A summary and discussion of 
different observational radius determinations can be found in [22]. In addition, for a rotating star due to its deformation there is no unambiguous relation between the observed quantity and the radii determined theoretically. On the theoretical side, due to the matching between a core and a crust EoS, not necessarily obtained within the same model, the calculated radii are subject to uncertainties of the order of several percent, too [63, 64]. However, much observational effort is being put into the determination of neutron star radii and further constraints are to be expected. Thus, it is interesting to investigate the radius range that neutron stars with hyperons can have.

The first remark to be made, looking at the radii in figure 3, is that the central density exceeds the threshold for the onset of $\Lambda$-hyperons only for neutron stars with $M \gtrsim 1.5 M_{\odot}$ (GM1) and $M \gtrsim 1.4 M_{\odot}(\mathrm{DDH} \delta)$, respectively. Thus the radius at the canonical mass of $M=1.4 M_{\odot}$ is almost exclusively determined by the nuclear part of the EoS, i.e. the nuclear interaction. The finding that the radius (see table 2 for a summary of the different values) for $M=1.4 M_{\odot}$ is significantly higher in GM1 than in DDH $\delta$ is consistent with it being dominated by the nuclear interaction and shows again the strong impact of the incompressibility and the symmetry energy $a_{\text {sym }}$ and its slope $L$, see table 1 , which are considerably lower in DDH $\delta$ than in GM1. It is, however, not true that hyperons cannot be added to nuclear models with low incompressibility or symmetry energy and slope without violating the neutron star maximum mass constraint, see the examples in $\mathrm{DDH} \delta$ in figure 3, see table 2. We will further discuss this point in section 4.2 upon including the full baryonic octet.

4.1.3. Strangeness content of neutron star matter. Does the stiffening of the EoS necessary to obtain maximum masses of at least $\sim 2 M_{\odot}$ reduce the hyperon content of neutron star matter finally excluding hyperons from neutron stars? The general recipe to increase the maximum mass is clear: add additional short range repulsion. If this is done mainly in the hyperon sector, then the strangeness content will be reduced. This is what happens upon decreasing $z$. In figure 4 we display the ratio of strangeness density with respect to baryon number density in GM1 (left panel) and $\mathrm{DDH} \delta$ (right panel) as a function of radius for the maximum mass configurations obtained earlier.

The general trend confirms the findings of [10], decreasing $z$ decreases the hyperon content of neutron star matter. There is, however, a point to add. The hyperon content, as can be seen from figure 4 is very sensitive to the attraction furnished by a coupling to $\sigma^{*}$. Of course, adding attraction reduces again the maximum mass such that the general trend is not modified: a higher maximum mass means globally fewer hyperons. But the absolute value of the hyperon content is strongly model dependent. And, as can be seen from the examples in figure 4 present observations are far from excluding hyperons from neutron stars.

\subsection{Including the full octet}

In the previous section, only $\Lambda$-hyperons have been considered. Although $\Lambda$-hyperons are the first to appear in neutron star matter for most interactions (in particular for models with a repulsive $\Sigma^{-}$potential in symmetric nuclear matter) and are in general the most abundant hyperons, this is of course not completely realistic. Therefore, we will now repeat the same analysis, but allowing a priori all particles of the baryon octet to have a nonzero density. In the following subsections we will first perform a stability analysis, and then look at neutron star properties. 

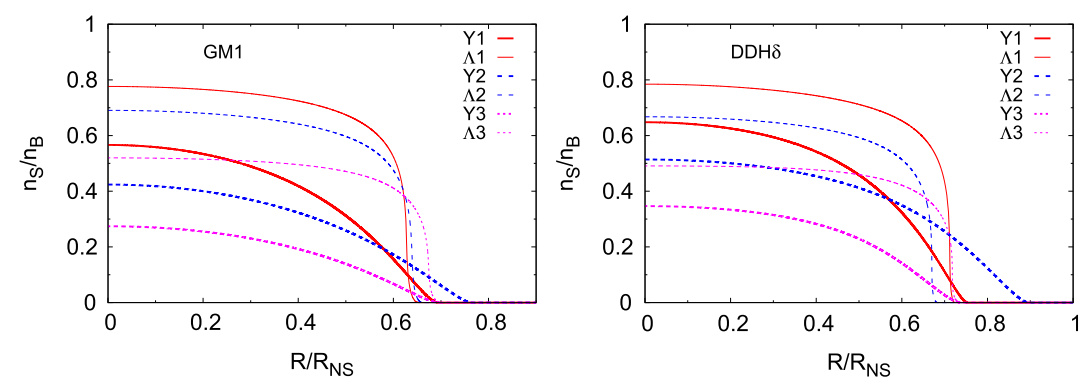

Figure 4. The ratio of strangeness density ( $=n_{\Lambda}$ for the present case) to baryon number density, $Y_{S}=n_{S} / n_{B}$ as a function of radius, normalized to the star's radius, for the respective maximum mass configuration of a spherical star. The GM1 (left) and DDH (right) parameter sets have been employed. The setup is the same as in figure 1.

4.2.1. Stability analysis. As in section 4.1.1 we are interested here in sets of couplings describing stellar matter with an instability at the onset of hyperons. For a given choice of the isoscalar vector meson couplings $g_{\phi i}$ and/or $g_{\omega i}$ and fixing the couplings to $\sigma$ by the values of the hyperon potentials in nuclear matter, the only remaining parameters are the couplings to $\sigma^{*}$. The isovector vector couplings are kept fixed by isospin symmetry.

In table 3 for GM1 and table 4 for TM1-2 and DDH $\delta$ we adopt the following convention: if no instability is present the model is identified with $Y n$; the sets identified with $\Lambda n$ all lead to an instability at the onset of $\Lambda$; the sets identified with $\Xi n$ and $\Sigma n$ originate an instability driven by the onset of the $\Xi$ or the $\Sigma$ isovector multiplet.

Let us start with the results respecting the symmetry constraints of equations (18). We will show only results obtained using GM1 here for two reasons. First, as discussed in section 4.1 qualitatively the results are similar for different models. Secondly, as shown in section 4.1.2, for the models including only the $\Lambda$-hyperon, the parameter space for obtaining high enough neutron star masses is very reduced within $\mathrm{DDH} \delta$ upon applying the symmetry constraints to the isoscalar vector couplings. The allowed parameter space becomes even smaller if the full octet is considered and the results do not give us any new insight with respect to those with GM1 presented here.

In figure 5 we show the minimal eigenvalue of the curvature matrix, $c_{\min }$, as a function of baryon number density in neutron star matter for different choices of the couplings to $\sigma^{*}$ and $z$ within GM1. The choice of couplings in the upper panel corresponds to those giving $U_{\Lambda}^{(\Lambda)}\left(n_{0} / 5\right)=-5 \mathrm{MeV}$ and $U_{\Xi}^{(\Xi)}\left(n_{0} / 5\right)=-10 \mathrm{MeV}$. Since only $\Lambda, \Xi^{-}$and $\Xi^{0}$-hyperons appear in neutron star matter with the employed parameter set, only the couplings associated to these hyperons are given. The $\Sigma$-hyperons, mainly due to the assumed strongly repulsive $\Sigma N$ interaction, appear only well above $n_{B}=1 \mathrm{fm}^{-3}$ beyond the central density of the neutron stars with the highest mass. In the curves, successive thresholds leading to kinks in $c_{\min }$ can be observed. They correspond to the onset of $\Lambda, \Xi^{-}$and $\Xi^{0}$-hyperons, respectively.

For the above choice of parameters, the system is perfectly stable. However, we have seen before for the case of neutron star matter with $\Lambda$-hyperons, see figure 1 , that increasing $g_{\sigma^{*} i}$ decreases the minimal eigenvalue of the curvature matrix leading finally to an instability. This can again be observed here. In the middle and bottom panels, results are displayed with the smallest value of $g_{\sigma^{*} \Lambda}$ and $g_{\sigma^{*} \Xi}$, respectively, leading to an instability for a given choice of the other couplings. As can be seen in the bottom panel, within GM1, for the critical value of $g_{\sigma^{*} \Xi}$, the system is not driven into an instability when the $\Xi^{-}$sets in, but the instability arises 


(

of $z$ and the relation in equations 18. The parametrization GM1 has been ector sector, meaning that the isovector couplings are fixed by the value $R_{*_{*}}$ and $R_{\sigma^{*} \Xi}$ for models $\Lambda n$ and $\Xi n$ thereby correspond to the critical values of these coupling constants for the onset of an instability in cold $\beta$ equilibrated neutron star matter, in the $\Lambda$ and $\Xi$ channel, respectively. The central energy density, baryon number density and strangeness fraction $Y_{s}=n_{S} / n_{B}$ are given for the maximum mass configuration. $f_{S}$ represents the integral of the strangeness fraction $Y_{s} / 3$ over the whole star for the maximum mass configuration, as in [10]. The potentials $U_{\Lambda}^{(\Lambda)}, U_{\Xi}^{(\Xi)}$ and $U_{\Sigma}^{(\Sigma)}$ are calculated at $n_{0} / 5$.

\begin{tabular}{|c|c|c|c|c|c|c|c|c|c|c|c|c|c|c|}
\hline Model & $R_{\sigma^{*} \Lambda}$ & $R_{\sigma^{*} \Xi}$ & $R_{\sigma^{*} \Sigma}$ & $z$ & $\begin{array}{l}M_{\max } \\
\left(M_{\odot}\right)\end{array}$ & $\begin{array}{l}R_{1.4} \\
(\mathrm{~km})\end{array}$ & $\begin{array}{l}R_{\max } \\
(\mathrm{km})\end{array}$ & $\begin{array}{c}\epsilon^{(c)} \\
\left(\mathrm{MeVfm}^{-3}\right)\end{array}$ & $\begin{array}{c}n_{B}^{(c)} \\
\left(\mathrm{fm}^{-3}\right)\end{array}$ & $Y_{S}^{(c)}$ & $f_{S}$ & $U_{\Lambda}^{(\Lambda)}$ & $\begin{array}{c}U_{\Xi}^{(\Xi)} \\
(\mathrm{MeV})\end{array}$ & $\begin{array}{c}U_{\Sigma}^{(\Sigma)} \\
(\mathrm{MeV})\end{array}$ \\
\hline \multicolumn{15}{|l|}{ GM1 } \\
\hline$Y 4$ & 0.45 & 1.16 & 0 & 0.41 & 1.79 & 13.8 & 13.0 & 825.8 & 0.73 & 0.70 & 0.04 & -5 & -10 & 14 \\
\hline$\Lambda 4$ & 0.91 & 1.16 & 0 & 0.41 & 1.59 & 13.8 & 13.6 & 598.3 & 0.56 & 0.59 & 0.006 & -19 & -10 & 14 \\
\hline$\Xi 1$ & 0.45 & 1.35 & 0 & 0.41 & 1.71 & 13.8 & 13.4 & 737.1 & 0.67 & 0.75 & 0.02 & -5 & -21 & 14 \\
\hline$Y 5$ & 0.20 & 0.87 & 0 & 0.2 & 2.12 & 13.8 & 12.3 & 1040 & 0.85 & 0.71 & 0.07 & -5 & -10 & 14 \\
\hline$\Lambda 5$ & 1.0 & 0.87 & 0 & 0.2 & 1.94 & 13.8 & 11.0 & 491.1 & 1.08 & 0.93 & 0.20 & -27 & -10 & 14 \\
\hline$\Xi 2$ & 0.20 & 1.26 & 0 & 0.2 & 1.98 & 13.8 & 13.1 & 806.9 & 0.71 & 0.84 & 0.03 & -5 & -29 & 14 \\
\hline$Y 6$ & 0 & 0.55 & 0 & 0. & 2.29 & 13.8 & 12.1 & 1075 & 0.85 & 0.46 & 0.04 & -7 & -10 & 13 \\
\hline$\Lambda 6$ & 1.08 & 0.55 & 0 & 0. & 2.19 & 13.8 & 11.7 & 1139 & 0.90 & 0.64 & 0.12 & -34 & -10 & 13 \\
\hline$\Xi 3$ & 0 & 1.13 & 0 & 0. & 2.24 & 13.8 & 12.4 & 1004 & 0.82 & 0.76 & 0.05 & -7 & -33 & 13 \\
\hline
\end{tabular}


Table 4. Summary of results calculated within different models allowing for a free variation of the isoscalar vector couplings. The parameters used are indicated in columns 3-7. $R_{\omega \Lambda}$ and $R_{\phi \Lambda}$ represent the ratio of the corresponding isoscalar vector coupling constants to their respective $S U(6)$ values. As before, the couplings to $\sigma^{*}$ are defined with respect to $g_{\sigma N}$. The choices of parameters named $\Lambda n, \Xi n, \Sigma n$ with $g_{\sigma^{*} \gamma} \neq 0$ originate an instability driven by the onset of $\Lambda, \Xi$ or $\Sigma$, respectively. The parametrizations named $Y n$ do not show any instability. The central energy density and baryon number density are given for the maximum mass configuration. $f_{S}$ represents the integral of the strangeness fraction $Y_{s} / 3$ over the whole star for the maximum mass configuration. The potentials $U_{\Lambda}^{(\Lambda)}, U_{\Xi}^{(\Xi)}$ and $U_{\Sigma}^{(\Sigma)}$ are calculated at $n_{0} / 5$.

\begin{tabular}{|c|c|c|c|c|c|c|c|c|c|c|c|c|c|c|c|}
\hline Model & $\begin{array}{l}L \\
(\mathrm{MeV})\end{array}$ & $R_{\sigma^{*} \Lambda}$ & $R_{\sigma^{*} \Xi}$ & $R_{\sigma^{*} \Sigma}$ & $R_{\omega Y}$ & $R_{\phi Y}$ & $\begin{array}{l}M_{\max } \\
\left(M_{\odot}\right)\end{array}$ & $\begin{array}{l}R_{1.4} \\
(\mathrm{~km})\end{array}$ & $\begin{array}{l}R_{\max } \\
(\mathrm{km})\end{array}$ & $\begin{array}{c}\epsilon^{(c)} \\
\left(\mathrm{MeVfm}^{-3}\right)\end{array}$ & $\begin{array}{c}n_{B}^{(c)} \\
\left(\mathrm{fm}^{-3}\right)\end{array}$ & $f_{s}$ & $\begin{array}{c}U_{\Lambda}^{(\Lambda)} \\
(\mathrm{MeV})\end{array}$ & $\begin{array}{c}U_{\Xi}^{(\Xi)} \\
(\mathrm{MeV})\end{array}$ & $\begin{array}{c}U_{\Sigma}^{(\Sigma)} \\
(\mathrm{MeV})\end{array}$ \\
\hline \multicolumn{16}{|l|}{ TM1-2 } \\
\hline Y1 & 110 & 0 & 0 & 0 & 1 & 1 & 1.95 & 14.55 & 12.57 & 1028 & 0.86 & 0.15 & 1.7 & 21.1 & 16.17 \\
\hline $\mathrm{Y} 2$ & 55 & 0 & 0 & 0 & 1 & 1 & 1.94 & 13.43 & 12.02 & 1085 & 0.91 & 0.12 & 1.7 & 21.1 & 16.17 \\
\hline$\Lambda 1$ & 110 & 1.01 & 1 & 1 & 1 & 1 & 1.51 & 14.55 & 14.48 & 505.2 & 0.48 & 0.004 & -21.8 & -2.3 & -7.17 \\
\hline$\Lambda 2$ & 110 & 1.23 & 1.23 & 1.23 & 1 & 1.5 & 1.74 & 14.55 & 10.61 & 1634 & 1.28 & 0.40 & -23.8 & 23.1 & -0.84 \\
\hline$\Lambda 3$ & 110 & 1.48 & 1.48 & 1.48 & 1 & 2 & 1.90 & 14.55 & 11.04 & 1458 & 1.15 & 0.31 & -27.0 & 59.1 & -12.62 \\
\hline$\Lambda 4$ & 110 & 1.68 & 1.68 & 1.68 & 1.5 & 2 & 2.13 & 14.55 & 12.20 & 1119 & 0.90 & 0.16 & -41.1 & 36.0 & -20.96 \\
\hline$\Lambda 5$ & 55 & 1.445 & 1.445 & 1.445 & 1 & 2 & 1.85 & 13.43 & 10.76 & 1482 & 1.17 & 0.26 & -23.9 & 62.1 & -9.40 \\
\hline$\Lambda 6$ & 55 & 1.58 & 1.58 & 1.58 & 1.5 & 2 & 2.09 & 13.43 & 11.85 & 1337 & 0.92 & 0.11 & -33.7 & 44.4 & -12.77 \\
\hline$\Xi 1$ & 110 & 0.3 & 1.42 & 1 & 1 & 1 & 1.75 & 14.63 & 14.08 & 722.2 & 0.65 & 0.04 & -0.37 & -25.82 & -7.17 \\
\hline$\Xi 2$ & 55 & 0.3 & 1.42 & 1 & 1 & 1 & 1.78 & 13.43 & 12.89 & 921.5 & 0.78 & 0.06 & -0.37 & -25.26 & -6.93 \\
\hline$\Sigma 1$ & 110 & 0.8 & 1 & 1.1 & 1 & 1.2 & 1.75 & 14.57 & 13.21 & 856.4 & 0.76 & 0.11 & -9.80 & 11.04 & -9.68 \\
\hline$\Sigma 2$ & 110 & 0.8 & 1 & 1.23 & 1 & 1.5 & 1.87 & 14.57 & 13.12 & 921.5 & 0.79 & 0.10 & -3.74 & 35.11 & -12.75 \\
\hline$\Sigma 3$ & 110 & 0.8 & 1 & 1.37 & 1.3 & 1.5 & 2.05 & 14.57 & 12.91 & 959.0 & 0.80 & 0.12 & -4.19 & 30.36 & -14.66 \\
\hline \multicolumn{16}{|l|}{$\mathrm{DDH} \delta$} \\
\hline Y4 & 44 & 1.03 & 0 & 0 & 1.5 & 0.85 & 2.05 & 12.7 & 11.2 & 1217 & 0.99 & 0.04 & -5 & 79 & 62 \\
\hline Y5 & 44 & 1.03 & 1 & 1 & 1.5 & 0.85 & 2.00 & 12.7 & 11.1 & 1262 & 1.02 & 0.06 & -5 & 41 & 24 \\
\hline Y6 & 44 & 1.81 & 2.70 & 1.99 & 1.5 & 1.59 & 2.01 & 12.7 & 10.9 & 1325 & 1.06 & 0.08 & -0.2 & -1 & -0.4 \\
\hline$\Lambda 4$ & 44 & 1.5 & 0 & 0 & 1.5 & 0.85 & 2.04 & 12.7 & 10.9 & 1358 & 1.07 & 0.08 & -48 & 79 & 62 \\
\hline$\Xi 1$ & 44 & 1.81 & 2.85 & 1.99 & 1.5 & 1.59 & 2.00 & 12.7 & 10.9 & 1325 & 1.06 & 0.09 & -0.2 & -33 & -0.4 \\
\hline
\end{tabular}




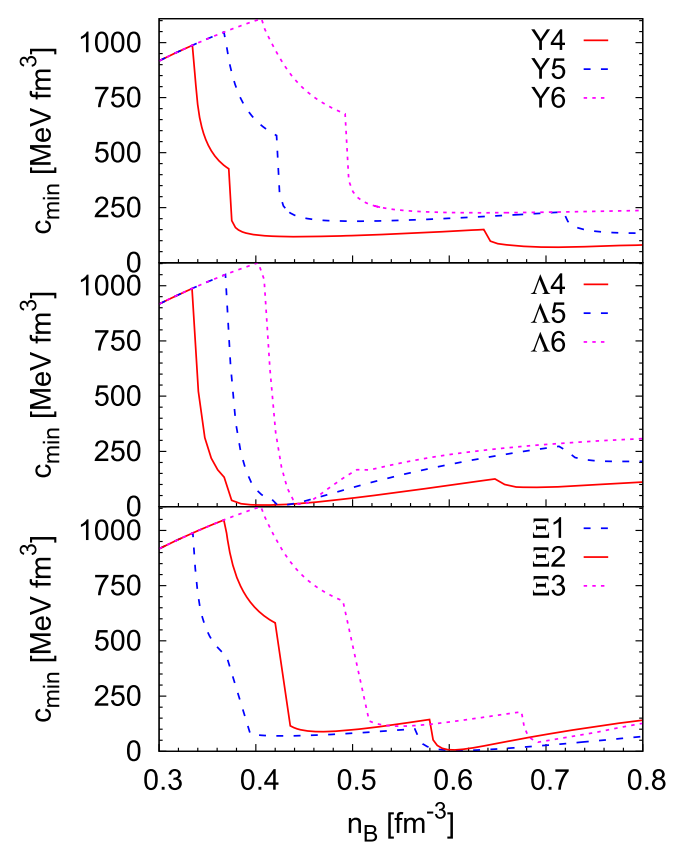

Figure 5. Smallest eigenvalue of the curvature matrix of the energy density as a function of baryon number density for neutron star matter in GM1 for different parameter sets including the full baryonic octet. Top: parameter sets not showing any instability; middle: instability driven by the onset of $\Lambda$; bottom: instability driven by the onset of $\Xi$. Parameters and neutron star properties are given in table 3 .

rather at the $\Xi^{0}$-threshold. In addition, although the $\Lambda$ is the first hyperon to appear, after the onset of $\Xi^{-}$, the number of $\Lambda$-hyperons remains almost constant with increasing density and even starts to decrease with the onset of $\Xi^{0}$ due to the large attraction that $\Xi^{-}$and $\Xi^{0}$ feel induced by the large coupling to $\sigma^{*}$. Changing the isovector channel by choosing a smaller symmetry energy slope would move the instability to larger densities, because a smaller $L$ disfavors the onset of neutral hyperons [29]. We will discuss this statement in a more quantitative way within two versions of TM1-2 below.

The critical values for $g_{\sigma^{*} \Lambda}$ leading to an instability are slightly lower than those obtained in section 4.1 .1 for $n, p, \Lambda, e$-matter, except for the $z=0$ case. At first sight this might be surprising since at densities below the $\Xi^{-}$-threshold, the results should be exactly the same. The reason is that actually the instability is not caused here by the onset of $\Lambda$-hyperons, but $\Xi^{-}$-hyperons. The threshold densities are very close and the two distinct thresholds are hardly distinguishable, looking at $c_{\min }$. A closer inspection of the data shows that the minimal value of $c_{\min }$ lies at densities above the $\Xi^{-}$-threshold, see figure 9 , too, where the number fractions are shown for the different species.

Not surprisingly, we are able to find an instability in other models, too. In figure 6 several examples of parameter sets leading to an instability within TM1-2 and DDH $\delta$ are shown. The $g_{\sigma Y}$ couplings are adjusted to the hyperon potentials in nuclear matter as before. Again, the values of $g_{\sigma^{*} i}$ correspond to the limiting values for the onset of an instability, as seen from the behavior of $c_{\min }$. No symmetry constraints have been imposed on the isoscalar vector couplings. All parameter values are listed in table 4. 


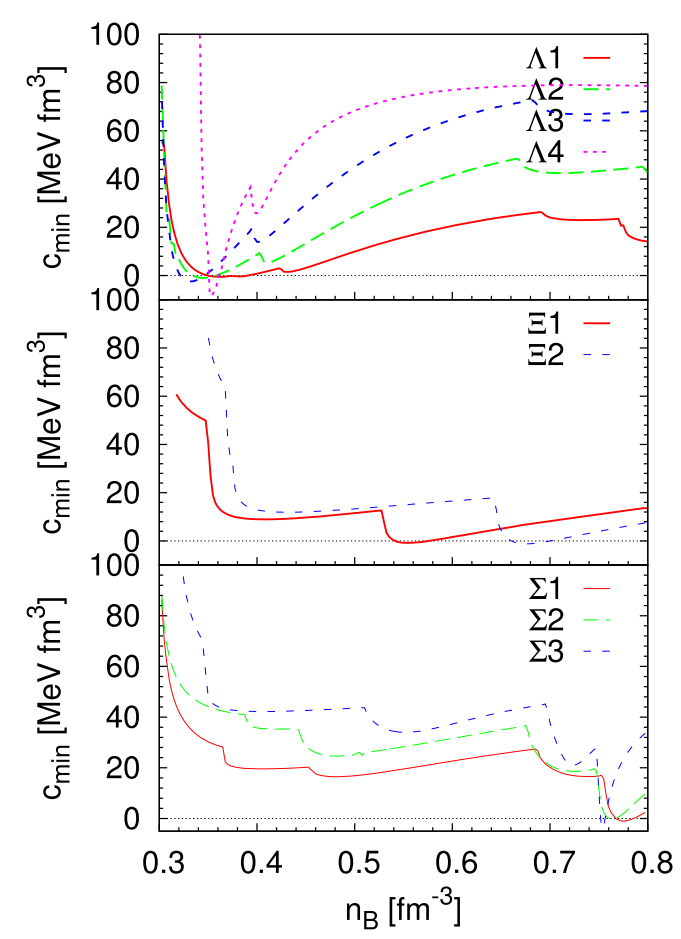

Figure 6. Smallest eigenvalue of the curvature matrix of the energy density as a function of baryon number density for neutron star matter in TM1-2 for different parameter sets including the full baryonic octet. Top: parameter sets with an instability driven by the onset of $\Lambda$; middle: instability driven by the onset of $\Xi$; bottom: instability driven by the onset of $\Sigma$. Parameters and neutron star properties are given in table 4 .

The sets in table 4 identified with $\Lambda n, \Xi n$ or $\Sigma n$, displayed in figure 6 in the top, middle and bottom panels, respectively, all lead to an instability at the onset of $\Lambda, \Xi$ or $\Sigma$ hyperons. A $\Xi$-driven instability is possible for strong $g_{\sigma^{*} \Xi}$ and weak $g_{\sigma^{*} \Lambda}$ couplings. A $\Sigma$-driven instability requires a strong $g_{\sigma^{*} \Sigma}$ coupling. Just as with GM1, a $\Xi$ or $\Sigma$-driven instability was only obtained after all members of the multiplet set in. It should be pointed out that, although the $\Sigma$ driven instability occurs at quite a high density, it still occurs within the range of densities inside a neutron star, see sections 4.2.2 and 4.2.3. A less repulsive $U_{\Sigma}^{(N)}$ would allow for an instability at lower densities. In the same way a less attractive $U_{\Xi}^{(N)}$ would remove the onset of the $\Xi$ inside a neutron star.

Let us mention that, in contrast to GM1, neutron star matter contains $\Sigma$-hyperons for some sets in table 4 with $U_{\Sigma}^{(N)}=30 \mathrm{MeV}$ and more than three thresholds can be observed within the range of densities relevant for neutron stars. We will discuss the composition in detail in section 4.2.3.

Of course, the $Y Y$-interaction is very sensitive to the couplings to $\sigma^{*}$. Remember that originally the $\sigma^{*}$ was introduced to allow for very attractive $Y Y$-interactions in view of experimental results for double- $\Lambda$ hypernuclei at the epoch in [49]. A strong attraction in the $\Lambda \Lambda$-channel was indeed found in section 4.1.1 for the values of $g_{\sigma^{*} \Lambda}$ leading to an instability. The same is true here, as seen for the $\Xi \Xi$-potentials and the $\Sigma \Sigma$-potentials, shown in figure 7 , right panel, and the $\Lambda \Lambda$-potential displayed in figure 7 , left panel, for different examples in TM1-2 and DDH $\delta$. The corresponding values at $n_{0} / 5$ are listed in table 4 . It is obvious that 

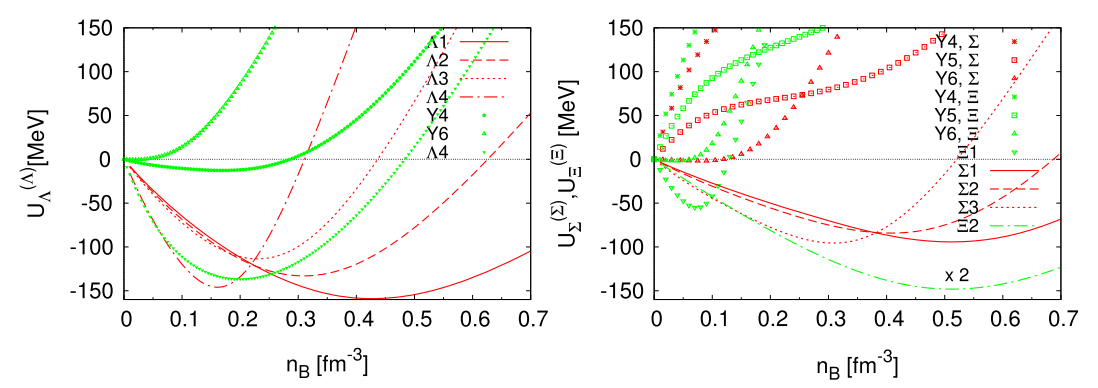

Figure 7. The $Y$-potential in isospin symmetric $Y$-matter as a function of baryon number density, for $\Lambda$ (left), $\Sigma$ and $\Xi$ (right) hyperons for a selection of parameter sets in table 4 as predicted by TM1-2 (lines) and DDH $\delta$ (symbols). The results for model $\Xi 2$ should be considered multiplied by a factor of 2 .

the attraction needed in the $\Lambda \Lambda$-channel to obtain an instability is much higher for all examples shown than the values suggested by experimental data. Even if we neglect the Nagara event and only consider the earlier data (see e.g. [49] for a discussion) resulting in a stronger attraction in this channel, the coupling strength needed for the onset of an instability is far off.

In the $\Xi \Xi$-channel and the $\Sigma \Sigma$-channel the situation is less evident because there is no experimental information available in these channels. Current information, based on theoretical arguments for the baryon octet in vacuum and corresponding meson exchange models, is clearly not sufficient to pin down the amount of attraction for the $Y Y$ interaction in dense matter. Since the coupling to $\sigma^{*}$ is determined mainly via the $Y Y$ interaction, more data, in particular on hyperons other than $\Lambda$-hyperons, would be very welcome to be able to judge whether the different chosen values are pertinent or not.

4.2.2. Neutron star masses and radii. In section 4.1 .2 we have presented results for neutron star masses and radii with matter containing neutrons, protons, $\Lambda$-hyperons and electrons. The conclusions were that, within this restricted setup, firstly the observed neutron star masses can not be used to exclude the existence of a first order phase transition to hyperonic matter in RMF models. Secondly, the radii for intermediate mass neutron stars are most sensitive to the properties of the nuclear interaction and, in contrast to previous claims, it is possible to obtain masses in agreement with recent observations for models containing a substantial amount of $\Lambda$-hyperons, using a nuclear interaction with low $L$ leading to relatively small radii of the order $12-13 \mathrm{~km}$ at intermediate masses.

Within this section we would like to investigate whether these conclusions about including the full baryonic octet remain true. From the simple argument that new degrees of freedom soften the equation of state we would expect the mass constraint to become more difficult to fulfil, at least if more than one hyperon species becomes populated. This argument is, however, only strictly valid for free Fermi gases without interaction and we thus have to study the questions within different interaction models.

In tables 3 (GM1) and 4 (TM1-2 and $\mathrm{DDH} \delta$ ) the maximum mass, the radius at a gravitational mass of $1.4 M_{\odot}$ and the radius at maximum mass for spherical non-rotating neutron stars are given for the different parameter sets discussed in the preceding section. In figure 8 the corresponding mass-radius relations are plotted, for TM1-2 in the left panel and GM1 and $\mathrm{DDH} \delta$ in the right panel. 

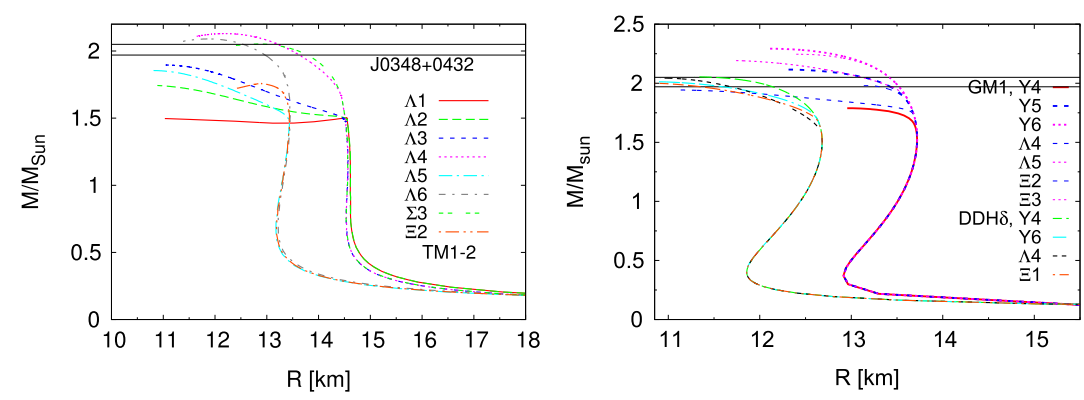

Figure 8. (Gravitational) mass/radius curves for spherical neutron stars for a selected choice of parameters using TM1-2 (left) and GM1/DDH $\delta$ (right). For TM1-2, we show the families of stars obtained with the parametrizations of table 4 that drive the stellar matter into an instability upon the onset of $\Lambda$. The two horizontal lines indicate the mass of PSR J0348 + 0432, $2.01 \pm 0.04 M_{\odot}$.

Very generally, increasing the magnitude of the vector-meson couplings allows for larger masses due to the stronger repulsion. This can be observed for all cases. Within GM1, respecting the symmetries, the maximum mass increases with decreasing $z$, see table 3 . For TM1 -2 and DDH $\delta$ no constraint is set on the isoscalar vector couplings and the maximum neutron star mass increases upon increasing those values, see table 4 .

Increasing the $\sigma^{*}$-couplings, leading to a stronger attraction, the maximum mass decreases. This can again be observed within all the models discussed here. For instance, taking for the isoscalar vector couplings their respective $S U(6)$ values, and choosing $g_{\sigma^{*} i}=0$, a maximum mass of $\sim 1.95 M_{\odot}$ is obtained for both $L=110$ and $55 \mathrm{MeV}$ within TM1-2. Increasing $g_{\sigma^{*} i}$ reduces the maximum mass as expected, and for $g_{\sigma^{*} A}$ crit $=1.01 g_{\sigma N}$ the maximum mass is $1.51 M_{\odot}$. If, however, no constraint is set on the isoscalar vector couplings, it is possible to choose a set of couplings which predict larger maximum masses, also above 2 $M_{\odot}$, and still give rise to an instability driven by the onset of strangeness.

Compared with the case of $n p \Lambda+e$-matter, see section 4.1.2, the maximum masses are reduced by the presence of other hyperon species, as expected. The effect is more pronounced if more species enter and if their respective threshold densities are considerably below the central density of the maximum mass configuration. For instance, as can be observed from the GM1 results, for the highest value of $z$ the maximum mass is strongly reduced with respect to the $n p \Lambda+e$ case and only very small for $z=0$. The reason is that for the respective maximum mass configurations, $\Xi^{0}$-hyperons enter for $z=0.41$ and $z=0.2$ in addition to $\Lambda$ and $\Xi^{-}$, whereas they are absent for the $z=0$ models, see figure 9, too. Another point should be mentioned concerning these results: the effect of increasing the attraction due to a $\sigma^{*}$-coupling between the canonical and the critical value is more pronounced for the threshold to $\Lambda$-hyperons than for the $\Xi$. The reason is that the difference between the canonical and the critical value in the $\Xi$-channel is smaller than for the $\Lambda$-hyperons, partly because at the $\Xi$ thresholds other hyperons are already present, pushing the instability.

From all the above-discussed examples, it is clear that the existence of an instability is not excluded by the neutron star maximum masses. The maximum masses are more strongly dependent on the vector couplings than on the $\sigma^{*}$-couplings for values between zero and the critical values, such that the allowed parameter space is still large. In addition, as discussed in section 4.1.2, there is no evidence that nuclear EoS with large $L$ have to be chosen to obtain maximum masses above $2 M_{\odot}$ with a considerable amount of hyperons in the central part. 

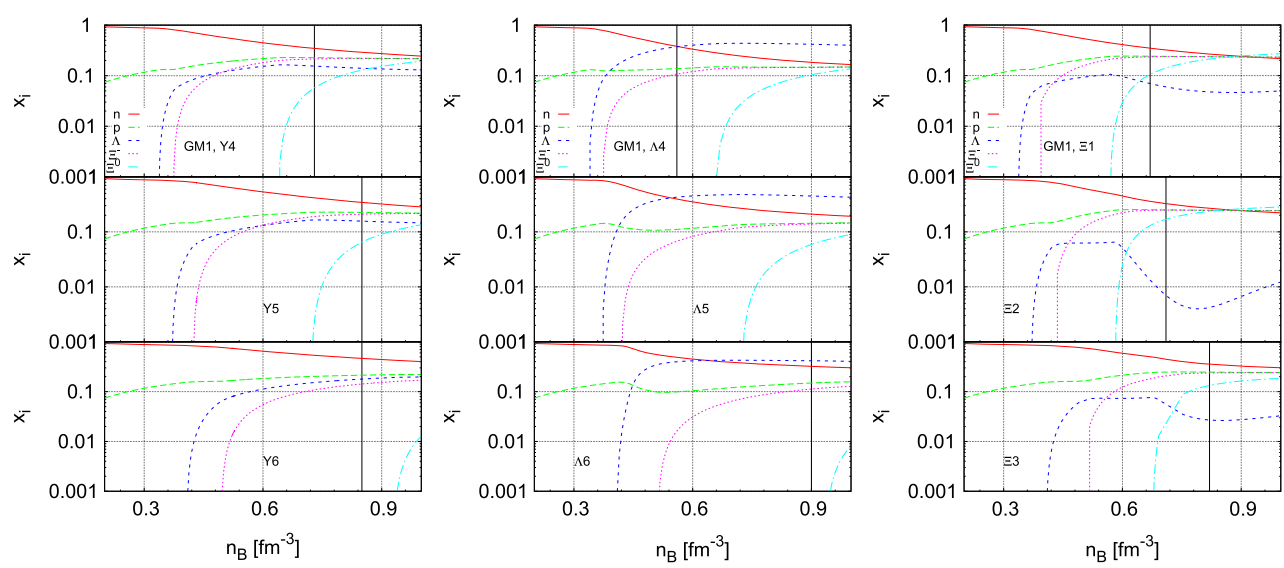

Figure 9. Particle fractions in neutron star matter $\left(x_{i}=n_{i} / n_{B}\right)$ for the different parameter sets discussed above within GM1. The vertical lines indicate the central density of the respective maximum mass configurations.

Since for almost all the parameter sets considered here, $\Lambda$-hyperons appear first, the neutron star radii at intermediate masses still depend mainly on the properties of the purely nuclear EoS and considerable differences due to the presence of hyperons can be observed only for masses close to the respective maximum mass. For the EoS giving acceptable maximum masses, the difference in radii due to hyperons becomes clearly visible for stars with masses above roughly $1.8 M_{\odot}$. Therefore, again, the main parameter determining the radii at intermediate masses is the slope of the symmetry energy, $L$, as found earlier in the context of purely nuclear models [35-37].

To illustrate this point, let us first compare the two versions of TM1-2. It should again be noted that within those models hyperons are present only in stars with $M \gtrsim 1.5 M_{\odot}$, such that the radius at $M=1.4 M_{\odot}$ is determined by the nuclear parameters. The original parametrization TM1-2 has a very large value of the symmetry energy slope at saturation $L$, and, not surprisingly the radius of a $1.4 M_{\odot}$ star is above $14 \mathrm{~km}$. However, including a nonlinear $\omega \rho$ term in the Lagrangian density it is possible to decrease $L$. For $L=55 \mathrm{MeV}$, a radius of $13.4 \mathrm{~km}$ is obtained. The DDH $\delta$ model has an even lower value of $L=44 \mathrm{MeV}$ and, as discussed already in section 4.1.2, the radius of a star with $M=1.4 M_{\odot}$ is $12.7 \mathrm{~km}$. Hyperons can be added to the EoS within this model without any contradiction to present neutron star mass observations.

Hence, since the maximum masses are only moderately influenced by the presence of hyperons other than $\Lambda^{\prime} \mathrm{s}$, we conclude as before that hyperons can be added to nuclear models with low symmetry energy and slope without violating the neutron star maximum mass constraint, and that in this way radii between $12-13 \mathrm{~km}$ can be obtained for neutron stars with the canonical mass of $1.4 M_{\odot}$, see the numbers given in table 4 .

4.2.3. Strangeness content of neutron star matter. In figure 9 we display the fractions of the different particles in neutron star matter as a function of baryon number density within GM1, varying $z$ and $g_{\sigma^{*} i}$. As expected, rendering the vector repulsion stronger by choosing a smaller value of $z$, the respective hyperonic thresholds are shifted to higher densities. Thereby, the cascade thresholds show a stronger $z$ dependence than the $\Lambda$-threshold. The reason is the stronger $z$-dependence of the individual hyperon-meson coupling constants, see 

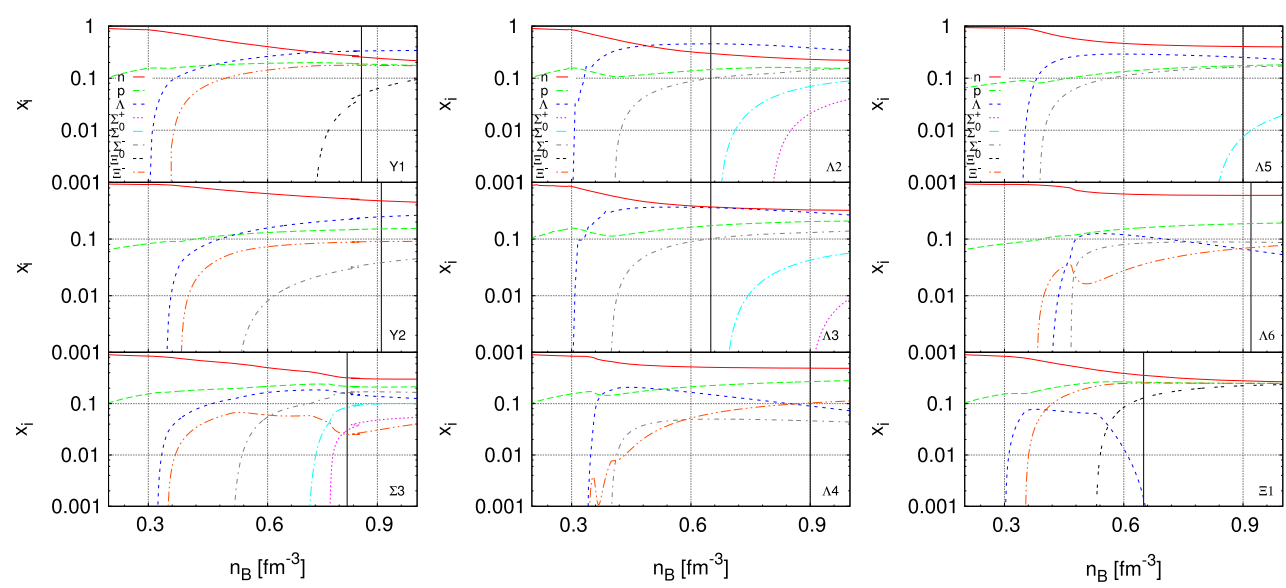

Figure 10. Particle fractions in neutron star matter $\left(x_{i}=n_{i} / n_{B}\right)$ for the different parameter sets discussed above within TM1-2. The vertical lines indicate the central density of the respective maximum mass configurations.

equation (18), induced by the symmetry requirements of the procedure used. It can be observed that all threshold densities for $z<0.41$ are significantly lower than those in [10], i.e., hyperons are present at much lower baryon number densities. This can be explained by a different readjustment of the couplings to $\sigma$ : we keep the values of the hyperon potentials in nuclear matter, $U_{\Lambda}^{(N)}\left(n_{0}\right)=-28 \mathrm{MeV}, U_{\Xi}^{(N)}\left(n_{0}\right)=-18 \mathrm{MeV}$ and $U_{\Sigma}^{(N)}\left(n_{0}\right)=30 \mathrm{MeV}$, constant when changing the value of $z$. In addition, all the curves have been calculated with nonzero coupling to $\sigma^{*}$.

The observed dependence on the attraction furnished by $\sigma^{*}$ is no surprise. Increasing $g_{\sigma^{*} i}$, the threshold density for hyperon $i$ is lowered and its abundance is globally increased. In particular, in the right panel it can be seen that for a strongly attractive coupling of $\Xi$ hyperons, although due to the much higher mass of the $\Xi$ with respect to $\Lambda$, the latter threshold still remains the lowest, at densities beyond the $\Xi^{0}$-threshold, $\Lambda$-hyperons become the less abundant ones. This is again an example which shows to what extent the composition of the core of neutron stars depends on the interactions between different particles and the necessity of more experimental data to pin down the neutron star composition.

The fractions of particles plotted in figure 10 were calculated within TM1-2 $(L=110 \mathrm{MeV})$ and TM1-2 with the $\omega \rho$ nonlinear term $(L=55 \mathrm{MeV})$. In case of an existing instability, it is driven by the hyperon indicated in the name of the parametrization, see table 4. Some additional comments are in order:

(a) for the hyperon potentials chosen and taking for the isoscalar vector mesons the $S U(6)$ couplings, the first hyperon to set in is always the $\Lambda$ followed by the $\Xi^{-}$;

(b) increasing the strength of the $g_{\phi Y}$ coupling with respect to its $S U(6)$ value disfavors the onset of $\Xi$ due to the large strangeness of these hyperons and the onset of $\Lambda$ is followed by the onset of $\Sigma^{-}$;

(c) increasing the strength of the $g_{\omega Y}$ coupling with respect to its $S U(6)$-value will disfavor more strongly the onset of $\Sigma$ and $\Lambda$, and, therefore, it may happen that the $\Xi^{-}$onset density is the lowest, mainly if $L=55 \mathrm{MeV}$, the latter having a larger $g_{\rho i}$ favoring negatively charged hyperons; 
(d) taking the smaller value of the symmetry energy slope, $L=55 \mathrm{MeV}$, the $\Lambda$-hyperons set in at larger densities, and the $\Sigma$-hyperons at smaller densities. The total strangeness inside the maximum mass star is smaller for the EoS with smaller $L$.

\section{Summary and conclusions}

We have investigated neutron star matter including hyperonic degrees of freedom within an RMF approach. For the nucleonic EoS we have considered the GM1 parametrization [28], the $\mathrm{DDH} \delta$ [31], and some variations of the TM1 parametrization [33] with a smaller symmetry energy slope $L$ and/or a harder EoS at large densities [29]. The hyperon-nucleon interactions have been adjusted to existing experimental data. Thereby we have followed two different strategies in the isoscalar vector sector: either symmetry constraints [39] have been imposed, relaxing the $S U(6)$-symmetry to fix the couplings as done in several recent works $[10,14,32]$ or no particular symmetry has been assumed between hyperonic and nuclear couplings. For the hyperon-hyperon interaction, that in the present formalism is described through the mesons with hidden strangeness, $\sigma^{*}$ and $\phi$, the couplings $g_{\sigma^{*} i}$ have been varied freely, and, in particular, they have been chosen strong enough to originate an instability with the onset of hyperons; whereas for the coupling to $\phi$, the prescription for the isoscalar vector sector has been followed.

Our main focus has been to study the possibility that an instability driven by the onset of hyperons could exist, the neutron star maximum mass, and the strangeness content of neutron star matter. We have also looked at the radii of intermediate mass neutron stars with EoS containing hyperons. The existence of an instability as a trace of a first order phase transition was identified by analyzing the curvature of the thermodynamical potential with respect to the baryonic, strangeness and leptonic densities. In all our studies at most one negative eigenvalue, corresponding to the direction in density space, in which density fluctuations are spontaneously and exponentially amplified in order to achieve phase separation, has been found.

First we have studied $n p \Lambda+e$ matter in $\beta$-equilibrium, and showed that it was possible to choose a set of parameters that gives rise to an instability driven by the onset of $\Lambda$ 's and still predict a maximum star mass of the order of $2 M_{\odot}$, and stars with a mass of $\sim 1.4 M_{\odot}$ with a radius of $12-13 \mathrm{~km}$. It was shown that the hyperon content is very sensitive to the attraction furnished by a coupling to $\sigma^{*}$ and that the absolute value of the hyperon content is strongly model dependent. The $n p e \Lambda+e$ calculations are far from excluding hyperons from neutron stars. The price to pay for having an instability is, however, a very strong $\Lambda-\Lambda$ attraction, which is in contradiction with the actual experimental information.

In a second step we considered the whole baryonic octet. Again, it was shown that a particular choice of the coupling parameters $g_{\sigma^{*} i}, g_{\phi i}$ and $g_{\omega i}$ allowed the construction of EoS giving rise to star masses as high as $2 M_{\odot}$, which, in addition, predict the occurrence of instabilities at the onset of hyperons. In particular, it was shown that it is possible to have an instability driven by the onset of the $\Lambda$-, the $\Xi$ - or the $\Sigma$-hyperons depending on the choice of the coupling parameters. The coupling parameters will also determine the different hyperon species and the strangeness fraction occurring inside a neutron star. Presently, the scarce amount of experimental information on the hyperon sector leaves too much freedom in adjusting the interaction parameters to give a definite answer about the composition of neutron star matter with hyperons. 
It was also shown that the neutron star radii at intermediate masses depend mainly on the properties of the purely nuclear EoS and considerable differences due to the presence of hyperons can be observed only for masses close to the respective maximum mass. The main parameter determining the radii at intermediate masses is the slope of the symmetry energy, $L$, as found earlier [35-37]. It was shown that hyperons can be added to nuclear models with low symmetry energy and slope without violating the neutron star maximum mass constraint, and that in this way radii between $12-13 \mathrm{~km}$ can be obtained for neutron stars with the canonical mass of $1.4 M_{\odot}$. From rather general arguments it seems indeed difficult to obtain even lower radii with an EoS satisfying the maximum mass constraint [65]. In fact, for almost all the parameter sets considered, the $\Lambda$-hyperons appear first, and the hyperons are present only in stars with $M \gtrsim 1.5 M_{\odot}$, such that the radius at $M=1.4 M_{\odot}$ is determined by the nuclear parameters. Contrary to existing models, see [22] for a discussion, we could get hyperonic stars described within a RMF calculation with a mass $M=2 M_{\odot}$ or above, and still satisfying the semi-empirical constraint on the pressure of neutron star matter at saturation density [38].

Our results obtained from a wide range of allowed parameter sets clearly show that additional constraints from future experimental data on hyperonic interactions and/or $a b$ initio calculations of baryonic matter with hyperons are necessary to clarify the structure and composition of neutron stars. Possible non-homogeneous phases in the star would affect the mean-free path of neutrinos (see e.g. [24]), and, consequently, the cooling rate of the star, or its transport properties, e.g. heat and electric conductivity, and shear viscosity.

A selection of the EoS presented in this paper is publicly available on the Compose web site [66] (http://compose.obspm.fr).

\section{Acknowledgments}

This work was partially funded by the SN2NS project ANR-10-BLAN-0503 and by Project PEst-OE/FIS/UI0405/2014 developed under the initiative QREN financed by the UE/FEDER through the program COMPETE/FCT, and was supported by NewCompstar, COST Action MP1304. ARR acknowledges partial support from the Romanian National Authority for Scientific Research under grants PN-II-ID-PCE-2011-3-0092 and PN 09370105 and kind hospitality from LPC-Caen and LUTH-Meudon.

\section{References}

[1] Glendenning N 1982 Phys. Lett. B114 392

[2] Demorest P B, Pennucci T, Ransom S M, Roberts M S and Hessels J W 2010 Nature 4671081

[3] Antoniadis J et al 2013 Science 3406131

[4] Zdunik J L and Haensel P 2013 Astron. Astrophys. 551 A61

[5] Drago A, Lavagno A, Pagliara G and Pigato D 2014 arXiv:1407.2843

[6] Hofmann F, Keil C M and Lenske H 2001 Phys. Rev. C 64025804

[7] Rikovska-Stone J, Guichon P A M, Matevosyan H H and Thomas A W 2007 Nucl. Phys. A 792341

[8] Bednarek I, Haensel P, Zdunik J L, Bejger M and Manka R 2012 Astron. Astrophys. 543 A157

[9] Weissenborn S, Chatterjee D and Schaffner-Bielich J 2012 Nucl. Phys. A 88162

[10] Weissenborn S, Chatterjee D and Schaffner-Bielich J 2012 Phys. Rev. C 85065802

[11] Bonanno L and Sedrakian A 2012 Astron. Astrophys. 529 A16

[12] Oertel M, Fantina A F and Novak J 2012 Phys. Rev. C 85055806

[13] Colucci G and Sedrakian A 2013 Phys. Rev. C 87055806

[14] Lopes L L and Menezes D P 2014 Phys. Rev. C 89025805

[15] Banik S, Hempel M and Bandyopadhyay D 2014 Astrophys. J. Suppl. 222214

[16] van Dalen E N E, Colucci G and Sedrakian A 2014 Phys. Lett. B 734383 
[17] Gomes R O, Dexheimer V, Schramm S and Vasconcellos C A Z 2014 arXiv: 1411.4875

[18] Vidana I, Logoteta D, Providencia C, Polls A and Bombaci I 2011 Europhys. Lett. 9411002

[19] Lonardoni D, Gandolfi S and Pederiva F 2013 Phys. Rev. C 87041303

Lonardoni D, Pederiva F and Gandolfi S 2014 Phys. Rev. C 89014314

[20] Lonardoni D, Lovato A, Gandolfi S and Pederiva F 2015 Phys. Rev. Lett. 114092301

[21] Katayama T and Saito K 2014 arXiv:1410.7166

[22] Fortin M, Zdunik J L, Haensel P and Bejger M 2015 Astron. Astrophys. 576 A68

[23] Gulminelli F, Raduta A R and Oertel M 2012 Phys. Rev. C 86025805

[24] Gulminelli F, Raduta A R, Oertel M and Margueron J 2013 Phys. Rev. C 87055809

[25] Raduta A R, Gulminelli F and Oertel M 2014 arXiv:1406.0395

[26] Schaffner-Bielich J and Gal A 2000 Phys. Rev. C 62034311

[27] Dutra M et al 2014 Phys. Rev. C 900552003

[28] Glendenning N K and Moszkowski S A 1991 Phys. Rev. Lett. 672414

[29] Providência C and Rabhi A 2013 Phys. Rev. C 87055801

[30] Avancini S S et al 2009 Phys. Rev. C 79035804

[31] Gaitanos T et al 2004 Nucl. Phys. A 73224

[32] Miyatsu T, Cheoun M-K and Saito K 2013 Phys. Rev. C 88015802

[33] Sugahara Y and Toki H 1994 Nucl. Phys. A 579557

[34] Danielewicz P, Lacey R and Lynch W G 2002 Science 2981592

[35] Horowitz C J and Piekarewicz J 2001 Phys. Rev. C 64062802

[36] Carriere J, Horowitz C J and Piekarewicz J 2003 Astrophys. J. 593463

[37] Cavagnoli R, Menezes D P and Providência C 2011 Phys. Rev. C 84065810

[38] Hebeler K, Lattimer J M, Pethick C J and Schwenk A 2013 Astrophys. J 77311

[39] Schaffner J and Mishustin I N 1996 Phys. Rev. C 531416

[40] Pile P H et al 1991 Phys. Rev. Lett. 662585

Hasegawa T et al 1996 Phys. Rev. C 531210

Hotchi H et al 2001 Phys. Rev. C 64044302

[41] Khaustov P et al 2000 Phys. Rev. C 61054603

[42] Saha P K et al 2004 Phys. Rev. C 70044613

Kohno M, Fujiwara Y, Watanabe Y, Ogata K and Kawai M 2006 Phys. Rev. C 74064613

[43] Nagae T et al 1998 Phys. Rev. Lett. 801605

[44] Vidana I, Polls A, Ramos A and Schulze H-J 2001 Phys. Rev. C 64044301

[45] Khan E et al 2014 private communication and in preparation

[46] Franklin G B 1995 Nucl. Phys. A $58583 \mathrm{c}$

[47] Aoki S et al 2009 Nucl. Phys. A 828191

[48] Ahn J K et al 2013 Phys. Rev. C 88014003

[49] Schaffner J et al 1994 Annals Phys. 23535

[50] Avancini S S, Brito L, Chomaz P, Menezes D P and Providência C 2006 Phys. Rev. C 74024317

[51] Ducoin C, Chomaz P and Gulminelli F 2006 Nucl. Phys. A 77168

[52] Ducoin C, Providência C, Santos A M, Brito L and Chomaz P 2008 Phys. Rev. C 78055801

[53] Ducoin C, Hasnaoui K H O, Napolitani P, Chomaz P and Gulminelli F 2007 Phys. Rev. C 75 065805

[54] Providência C, Brito L, Avancini S S, Menezes D P and Chomaz P 2006 Phys. Rev. C 73025805

[55] Gusakov M E, Haensel P and Kantor E M 2014 Mon. Not. R. Astron. Soc. 439318

[56] Rios A et al 2005 Phys. Rev. C 71055802

Lopez-Val D et al 2006 Phys. Rev. C 74068801

[57] Serot B D and Walecka J D 1997 Int. J. Mod. Phys. E 6515

[58] Brito L, Chomaz P, Menezes D P and Providência C 2007 Phys. Rev. C 76044316

[59] Tolman R C 1934 Proc. Nat. Sci. USA 20 169-76

Oppenheimer J R and Volkoff G M 1939 Phys. Rev. 55374

[60] Baym G, Pethick C and Sutherland P 1971 Astrophys. J. 170299

[61] Guillot S, Servillat M, Webb N A and Rutledge R E 2013 Astrophys. J. 77229

[62] Heinke C O et al 2014 Mon. Not. R. Astron. Soc. 444 443-56

[63] Ishizuka C and Ohnishi A 2014 Presentation at NICXIII

[64] Providência $\mathrm{C}$ in preparation

[65] Kurkela A, Fraga E S, Schaffner-Bielich J and Vuorinen A 2014 Astrophys. J. 789127

[66] Typel S, Oertel M and Klähn T 2013 arXiv:1307.5715 